\title{
Finite Section Method in some Algebras of Multiplication and Convolution Operators and a Flip
}

\author{
S. Roch, P. A. Santos and B. Silbermann
}

\begin{abstract}
This paper is concerned with the applicability of the finite section method to operators belonging to the closed subalgebra of $\mathcal{L}\left(L^{2}(\mathbb{R})\right)$ generated by operators of multiplication by piecewise continuous functions in $\dot{\mathbb{R}}$, convolution operators - also with piecewise continuous generating functions - and the flip operator $(J u)(x)=u(-x)$. For this, a larger algebra of sequences is introduced, which contains the special sequences we are interested in. There is a direct relationship between the applicability of the finite section method for a given operator and the invertibility of the corresponding sequence in this algebra. Exploring this relationship, the methods of essentialization, localization and identification of the local algebras through construction of locally equivalent representations are used and so useful invertibility criteria are derived. Finally, examples are presented, including explicit conditions for the applicability of the finite section method to a Wiener-Hopf plus Hankel operator with piecewise continuous symbols, and some relations between the approximation operators and the limit operator are discussed.
\end{abstract}

Keywords: Finite section method, Wiener-Hopf operators, Hankel operators

AMS subject classification: $65 \mathrm{R} 20,47 \mathrm{C} 15$

\section{Introduction}

The study of canonical diffraction problems can be frequently reduced to a system of equations in $L^{2}(\mathbb{R})$, whose associated operator is of the form Wiener-Hopf plus Hankel operator, with piecewise continuous symbols (see, for instance, $[12,13,20]$ ). It is then of importance to be able to solve these systems of equations numerically (cf. [14]). However, it is known that the invertibility of the associated operator is not sufficient to guarantee convergence of the approximation methods normally used $[8,15]$. To identify the extra necessary conditions, and to obtain a set of sufficient conditions, the most effective method is to 'algebraize' the applicability of the approximation method (i.e. to reformulate the problem as one of invertibility in a Banach algebra), following an

S. Roch: Inst. Math. Univ., Augustusplatz 10 - 11, D - 04109 Leipzig e-mail: sroch@mathematik.tu-chemnitz.de; supported by a DFG-Heisenberg grant.

P. A. Santos: Techn. Univ. Chemnitz-Zwickau, Fac. Math., D - 09107 Chemnitz; supported by grant Praxis XXI/BD/4501/94 from JNICT.

B. Silbermann: Techn. Univ. Chemnitz.Zwickau, Fac. Mat., D - 09107 Chemnitz

ISSN 0232-2064 / S 2.50 (C) Heldermann Verlag Berlin 
idea by Kozak [10]. In [21] one of the authors managed for the first time to obtain necessary and sufficient conditions for the applicability of the finite section method to one-dimensional Toeplitz operators with piecewise continuous coefficients through the choice of a suitable algebra. In 1983 Boettcher and one of the authors [3] advanced the idea in the case of Toeplitz matrices of associating these extra conditions to a symbol. It happens that this idea is applicable to a great variety of cases (see, for instance, the books $[4,9,15]$ and the literature cited there). It should also be mentioned the earlier monograph of Gohberg and Feldman [8], which encouraged many people to work in this field of research.

In this paper we are concerned with the applicability of the finite section method to operators belonging to the closed subalgebra of $\mathcal{L}\left(L^{2}(\mathbb{R})\right)$ generated by operators of multiplication by piecewise continuous functions in $\dot{\mathbb{R}}$, convolution operators - also with piecewise continuous generating functions - and the flip operator $(J u)(x)=u(-x)$. The algebraization is made by introducing a larger algebra of sequences which contains the special sequences we are interested in. There is a direct relationship between the applicability of the finite section method for a given operator and the invertibility of the corresponding sequence in this algebra. Exploring this relationship, the methods of essentialization, localization and identification of local algebras through construction of locally equivalent representations are used, and so useful invertibility criteria are derived.

There exists the forerunner [16] to this paper written by one of the authors. In it, the case without the flip is treated for operators in $L^{p}(\mathbb{R}), 1<p<\infty$. But as that work is not readily available, and the sequence algebra introduced here is a little more general even in the case without the flip, we opted for a complete treatment of the case $p=2$ (with and without flip); also because it is now possible to add some results regarding spectral properties of the approximation sequences, which were not covered in the earlier work.

The present paper is organized as follows: Section 1 is the introduction which contains some definitions and relations that will be necessary. The main part is Section 2 , where criteria for the applicability of the finite section method are derived. In Section 3 some examples are presented, and finally, in Section 4 some relations between the approximation and limit operators are remarked.

In order not to burden the notation, we made the choice, when the meaning is clear, to represent by the same symbol a scalar operator and its generalization to the matrix case as a diagonal operator matrix, and a homomorphism defined on an algebra and the respective quotient homomorphisms defined on the quotient algebras (when they exist).

As usual, we let $L^{2}(\mathbb{R})$ represent the Hilbert space of Lebesgue integrable complexvalued functions $u$ defined in $\mathbb{R}$ such that the norm $\left(\int_{-\infty}^{+\infty}|u(x)|^{2} d x\right)^{\frac{1}{2}}$ is finite. The set of continuous functions defined on $\mathbb{R}$ - the one-point compactification of $\mathbb{R}$ - is represented by $C(\mathbb{R})$, and the set of piecewise functions on $\mathbb{R}$, that is, functions with well defined one-sided limits at all points of $\mathbb{R}$, by $P C(\dot{\mathbb{R}})$. Both these function sets are considered as subalgebras of $L^{\infty}(\mathbb{R})$. 
Denote by $F$ the Fourier transform defined from $L^{2}(\mathbb{R})$ onto itself:

$$
(F u)(y)=\int_{-\infty}^{+\infty} e^{i y x} u(x) d x
$$

and by $F^{-1}$ its inverse:

$$
\left(F^{-1} v\right)(x)=\frac{1}{2 \pi} \int_{-\infty}^{+\infty} e^{-i x y} v(y) d y
$$

For $a \in P C(\dot{\mathbb{R}})$ define in $L^{2}(\mathbb{R})$ the multiplication operator

$$
(a I u)(t)=a(t) u(t)
$$

and the convolution operator

$$
\grave{W}(a)=F^{-1} a F .
$$

By $\chi_{+}$and $\chi_{-}$there is represented the operator of multiplication by the characteristic function of the set $\mathbb{R}^{+}=(0,+\infty)$ and $\mathbb{R}^{-}=(-\infty, 0)$, respectively, and by $J$ the flip operator

$$
(J u)(t)=u(-t)
$$

Let $\tau$ be a positive real number, $I$ represent the identity operator and define the following operators acting on $L^{2}(\mathbb{R})$ :

$$
\begin{aligned}
\left(P_{\tau} u\right)(t) & = \begin{cases}u(t) & \text { if }|t|<\tau \\
0 & \text { if }|t|>\tau\end{cases} \\
Q_{\tau} & =I-P_{\tau} \\
\left(R_{\tau} u\right)(t) & = \begin{cases}u(\tau-t) & \text { if } 0<t<\tau \\
u(-\tau-t) & \text { if }-\tau<t<0 \\
0 & \text { if }|t|>\tau\end{cases} \\
\left(V_{\tau} u\right)(t) & = \begin{cases}0 & \text { if }|t|<\tau \\
u(t-\tau) & \text { if } t>\tau \\
u(t+\tau) & \text { if } t<-\tau\end{cases} \\
\left(V_{-\tau} u\right)(t) & = \begin{cases}u(t+\tau) & \text { if } t>0 \\
u(t-\tau) & \text { if } t<0 .\end{cases}
\end{aligned}
$$

The properties described in the next lemma of the above defined operators are very important and easily demonstrable.

Lemma 1.1. The following relations hold:

- $R_{\tau} P_{\tau}=P_{\tau} R_{\tau}=R_{\tau}, \quad P_{\tau}=R_{\tau}^{2}, R_{\tau}^{*}=R_{\tau},\left\|R_{\tau}\right\|=1$.

- $V_{\tau} V_{-\tau}=Q_{\tau}, V_{-r} V_{\tau}=I,\left(V_{r}\right)^{*}=V_{-\tau},\left\|V_{\tau}\right\|=\left\|V_{-r}\right\|=1$.

- $J V_{t r}=V_{t r} J, J R_{\tau}=R_{\tau} J$.

- $P_{\tau} \rightarrow I, V_{-\tau} \rightarrow 0$ strongly and $R_{\tau} \rightarrow 0, V_{\tau} \rightarrow 0$ weakly as $\tau \rightarrow \infty$. 


\section{Finite section in algebras generated by a shift, multiplication and convolution operators}

2.1 The finite section method. Consider the approximation method

$$
\left(P_{\tau} A P_{\tau}+Q_{r}\right) u_{\tau}=v
$$

to solve the equation

$$
A u=v \quad\left(A \in \mathcal{L}\left(L^{2}(\mathbb{R})\right) ; u, v \in L^{2}(\mathbb{R})\right)
$$

with $u_{r} \in L^{2}(\mathbb{R})$. This method is called the finite section method.

Definition 2.1. We say that the finite section method (1) applies to the operator $A \in \mathcal{L}\left({ }^{2}(\mathbb{R})\right)$ if

a) there exists a positive constant $\tau_{0}$ such that, for any $\tau>\tau_{0}$ and any $v$ in $L^{2}(\mathbb{R})$, there exists a unique solution $u_{\tau}$ of the equation $\left(P_{\tau} A P_{\tau}+Q_{\tau}\right) u_{\tau}=v$;

b) the sequence $\left(u_{r}\right)$ converges to a solution of the equation $A u=v$.

2.2 Algebraization. Let $\mathcal{E}$ be the set formed by all the sequences $\left(A_{\tau}\right)_{\tau \in \mathbb{R}^{+}}$of operators $A_{\tau}: L^{2}(\mathbb{R}) \rightarrow L^{2}(\mathbb{R})$ such that $\sup _{\tau}\left\|A_{\tau}\right\|_{\mathcal{L}_{\left(L^{2}\right)}}<\infty$. This set with the operations $\left(A_{\tau}\right)+\left(B_{\tau}\right)=\left(A_{\tau}+B_{\tau}\right)$ and $\left(A_{\tau}\right)\left(B_{\tau}\right)=\left(A_{\tau} B_{\tau}\right)$, with the norm $\left\|\left(A_{\tau}\right)\right\|=$ $\sup _{\tau}\left\|A_{\tau}\right\|_{\mathcal{L}\left(L^{2}\right)}$ and involution $\left(A_{\tau}\right)^{*}=\left(A_{\tau}^{*}\right)$ is a unital $C^{*}$-algebra. Note that the constant sequences $(A)$ are included in $\mathcal{E}$ for any $A \in \mathcal{L}\left(L^{2}\right)$.

Let $\mathcal{G}$ be the closed two-sided ideal in $\mathcal{E}$ of all sequences $\left(A_{T}\right)$ for which we have $\lim _{\tau \rightarrow \infty}\left\|A_{\tau}\right\|=0$.

Definition 2.2. We say that the sequence $\left(A_{\tau}\right) \in \mathcal{E}$ is stable if there exists a positive constant $\tau_{0}$ such that, for any $\tau>\tau_{0}, A_{\tau}$ is invertible and $\sup _{\tau>\tau_{0}}\left\|A_{\tau}^{-1}\right\|<\infty$.

The next result is then well known, but for the readers convenience we include a proof here.

Theorem 2.1. The following propositions are equivalent for $A_{\tau}=P_{\tau} A P_{\tau}+Q_{\tau}(A$ $\left.\in \mathcal{L}\left(L^{2}(\mathbb{R})\right)\right)$ :

a) The approximation method (1) applies to $A$.

b) $\left(A_{\tau}\right)$ is stable and $A$ is invertible.

c) $A$ is invertible, and the coset $\left(A_{r}\right)+\mathcal{G}$ is invertible in the quotient algebra $\mathcal{E} / \mathcal{G}$.

Proof. $a \Rightarrow b$ : Property a) implies that there exists a positive constant $\tau_{0}$ such that, for any $\tau>\tau_{0}, A_{\tau}$ is invertible and $A_{\tau}^{-1} v$ converges for any $v \in L^{2}$. By the Banach-Steinhaus theorem, $\sup _{\tau>\tau_{0}}\left\|A_{\tau}^{-1}\right\|<\infty$. To prove that $A$ is invertible note that

$$
\left\|u-A_{\tau}^{-1} A u\right\| \leq\left\|A_{\tau}^{-1}\right\|\left\|A_{\tau} u-A u\right\|
$$


the first factor of the right-hand side being uniformly bounded and the second going to zero as $\tau$ increases. So if $u \in \operatorname{ker} A$, we have immediately that $\|u\|=0$, and this means that $u=0$. So $\operatorname{ker} A=\{0\}$, and as $\operatorname{Im}\{A\}=L^{2}$ (by property a)), $A$ is invertible.

$b \Rightarrow c$ : Put $B_{\tau}=A_{\tau}^{-1}$ for $\tau>\tau_{0}$ and $B_{\tau}=I$ in the other cases. Then it is not dificult to see that

$$
\left(B_{\tau}\right)\left(A_{\tau}\right)=\left(A_{\tau}\right)\left(B_{\tau}\right) \in(I)+\mathcal{G} .
$$

$c \Rightarrow a$ : Let $\left(B_{r}\right)+\mathcal{G}$ be the inverse of $\left(A_{\tau}\right)+\mathcal{G}$. Then $B_{r} A_{\tau}=I+C_{\tau}$ and $A_{\tau} B_{\tau}=I+D_{\tau}$, with $C_{\tau}, D_{\tau} \in \mathcal{G}$. Choose $\tau_{0}$ such that $\left\|C_{\tau}\right\|<\frac{1}{2}$ and $\left\|D_{\tau}\right\|<\frac{1}{2}$ for $\tau>\tau_{0}$. Then $I+C_{\tau}$ and $I+D_{\tau}$ are invertible for $\tau>\tau_{0}$ and $\sup _{\tau>\tau_{0}}\left\|\left(I+C_{\tau}\right)^{-1}\right\| \leq 2$. The operators $A_{\tau}$ are thus two-sided invertible and this permits us to write

$$
A_{\tau} u_{\tau}=v \quad \Longleftrightarrow \quad B_{\tau} A_{\tau} u_{\tau}=B_{\tau} v \quad \Longleftrightarrow \quad u_{\tau}=\left(I+C_{\tau}\right)^{-1} B_{\tau} v .
$$

We only have to prove now that $\left\|u-u_{\tau}\right\|_{L^{2}} \rightarrow 0$ with $u=A^{-1} v$. This follows from

$$
\left\|u-u_{\tau}\right\|=\left\|u-\left(I+C_{\tau}\right)^{-1} B_{\tau} A u\right\| \leq\left\|\left(I+C_{\tau}\right)^{-1} B_{\tau}\right\|\left\|A_{\tau} u-A u\right\|,
$$

the last term going to zero because of the strong convergence $\lim A_{r}=A$, and the uniform boundedness of $B_{\tau}$

2.3 Essentialization. Let $\mathcal{F} \subset \mathcal{E}$ be the set of all sequences $\left(A_{r}\right)$ for which there exist operators $A$ and $A_{i j} \quad(i, j=1,2)$ such that the following strong limits as $\tau \rightarrow \infty$ exist (note that the arrow " $\rightarrow$ " between a sequence of operators and an operator will in this paper indicate strong convergence as $\tau$ goes to infinity):

- $A_{\tau} \rightarrow A$ and $A_{\tau}^{*} \rightarrow A^{*}$.

- $R_{\tau} A_{\tau} R_{\tau} \rightarrow A_{11}$ and $\left(R_{\tau} A_{\tau} R_{\tau}\right)^{*} \rightarrow A_{11}^{*}$.

- $R_{\tau} A_{\tau} V_{\tau} \rightarrow A_{12}$ and $\left(R_{\tau} A_{\tau} V_{\tau}\right)^{*} \rightarrow A_{12}^{*}$.

- $V_{-\tau} A_{\tau} R_{\tau} \rightarrow A_{21}$ and $\left(V_{-\tau} A_{\tau} R_{\tau}\right)^{*} \rightarrow A_{21}^{*}$.

- $V_{-\tau} A_{\tau} V_{\tau} \rightarrow A_{22}$ and $\left(V_{-\tau} A_{\tau} V_{\tau}\right)^{*} \rightarrow A_{22}^{*}$.

It should be remarked that the operators $A_{i j} \quad(i, j=1,2)$ do not depend in principle only on the operator $A$, but more on the sequence $\left(A_{r}\right)$. Using the fact that $I=$ $R_{\tau} R_{\tau}+V_{\tau} V_{-\tau}$ and Lemma 1.1 , one can see that this set is actually a closed $C^{*}$ subalgebra of $\mathcal{E}$ which contains $\mathcal{G}$.

Now let $\mathcal{K} \subset \mathcal{L}\left(L^{2}(\mathbb{R})\right)$ denote the ideal of compact operators and define $\mathcal{J}_{0}$ and $\mathcal{J}_{1}$ to be the sets

$$
\mathcal{J}_{0}=\left\{(K)+\left(G_{\tau}\right) \mid K \in \mathcal{K} \text { and }\left(G_{\tau}\right) \in \mathcal{G}\right\}
$$

and

$$
\mathcal{J}_{1}=\left\{\begin{array}{l|l}
\left(R_{\tau} K_{1} R_{\tau}+R_{\tau} K_{2} V_{-\tau}+V_{\tau} K_{3} R_{\tau}+V_{\tau} K_{4} V_{-\tau}\right)+\left(G_{\tau}\right) \mid \begin{array}{c}
K_{k} \in \mathcal{K} \\
\left(G_{\tau}\right) \in \mathcal{G}
\end{array}
\end{array}\right\}
$$

Proposition 2.2. $\mathcal{J}_{0}$ is a closed two-sided ideal of $\mathcal{F}$. 
Proof. It is clear that $\mathcal{J}_{0}$ is a linear subspace of $\mathcal{F}$. To prove that it is a left ideal it is enough to see that the sequences $\left(A_{T}\right)(K)$ are in $\mathcal{J}_{0}$. Writing

$$
\left(A_{\tau}\right)(K)=(A K)-\left(\left(A-A_{\tau}\right) K\right)
$$

one can see that the first term of the right-hand side is obviously in $\mathcal{J}_{0}$, and the second is even in $\mathcal{G}$ because $A_{\tau} \rightarrow A$ strongly as $\tau \rightarrow \infty$. To prove that $\mathcal{J}_{0}$ is a right ideal note that

$$
(K)\left(A_{\tau}\right)=(K A)-\left(K\left(A-A_{\tau}\right)\right)
$$

and $A_{r}^{*} \rightarrow A^{*}$. To prove that it is closed, remember first that if $\left(j_{r}\right)=\left(K+G_{r}\right)$, then as $j_{\tau}$ converges to $K$ in the norm, we have $\|K\|=\lim _{r \rightarrow \infty}\left\|K+G_{\tau}\right\|$. Then consider a Cauchy sequence in $\mathcal{J}_{0},\left(j_{r}\right)^{(k)}=\left(K^{(k)}+G_{\tau}^{(k)}\right)$, and because of the above result the sequence $K^{(k)}$ is also a Cauchy sequence, which means that there exists a compact operator $K$ such that $\left\|K-K^{(k)}\right\| \rightarrow 0$. But then $\left(G_{\tau}^{(k)}\right)$ is also a Cauchy sequence, and there exists $\left(G_{\tau}\right)$ such that $\left\|G_{T}-G_{T}^{(k)}\right\| \rightarrow 0$. We conclude that the sequence $\left(j_{\tau}\right)=\left(K+G_{\tau}\right)$ is the limit of $\left(j_{r}\right)^{(k)}$ and this ends the proof

\section{Proposition 2.3. $\mathcal{J}_{1}$ is a closed two-sided ideal of $\mathcal{F}$.}

Proof. We will only prove that $\mathcal{J}_{1}$ is a left ideal. The proof that it is a right ideal is similar. For $\left(A_{\tau}\right) \in \mathcal{F}$,

$$
\begin{aligned}
A_{\tau} R_{\tau} K_{1} R_{\tau}= & R_{\tau} R_{\tau} A_{\tau} R_{\tau} K_{1} R_{\tau}+V_{\tau} V_{-\tau} A_{\tau} R_{\tau} K_{1} R_{\tau} \\
= & R_{\tau} A_{11} K_{1} R_{\tau}+R_{\tau}\left(R_{\tau} A_{\tau} R_{\tau}-A_{11}\right) K_{1} R_{\tau} \\
& +V_{\tau} A_{21} K_{1} R_{\tau}+V_{\tau}\left(V_{-\tau} A_{\tau} R_{\tau}-A_{21}\right) K_{1} R_{\tau}
\end{aligned}
$$

the sequence corresponding to the second and fourth terms being in the ideal $\mathcal{G}$ due to the strong limits and the presence of the compact operator $K_{1}$. In a similar way we have

$$
\begin{gathered}
A_{\tau} R_{\tau} K_{2} V_{-\tau}=R_{\tau} A_{11} K_{2} V_{-\tau}+V_{\tau} A_{21} K_{2} V_{-\tau}+G_{\tau}^{(1)} \\
A_{\tau} V_{\tau} K_{3} R_{\tau}=R_{\tau} A_{12} K_{3} R_{\tau}+V_{\tau} A_{22} K_{3} R_{\tau}+G_{\tau}^{(2)} \\
A_{\tau} V_{\tau} K_{4} V_{-\tau}=R_{\tau} A_{12} K_{4} V_{-\tau}+V_{\tau} A_{22} K_{4} V_{-\tau}+G_{\tau}^{(3)}
\end{gathered}
$$

with $\left(G_{r}^{(k)}\right) \in \mathcal{G}$, and this proves that $\mathcal{J}_{1}$ is a left ideal. The closedness proof is as in Proposition 2.2, taking into account the existence of the strong limits of the algebra definition, in order to apply the Banach-Steinhaus theorem

We can now define the following *-homomorphisms in $\mathcal{F}$ :

$$
W^{0}: \mathcal{F} \rightarrow \mathcal{L}\left(L^{2}\right), \quad W^{0}\left(\left(A_{\tau}\right)\right)=A
$$

and

$$
W^{1}: \mathcal{F} \rightarrow \mathcal{L}\left(L^{2}\right)^{2 \times 2}, \quad W^{1}\left(\left(A_{\tau}\right)\right)=\left[\begin{array}{ll}
A_{11} & A_{12} \\
A_{21} & A_{22}
\end{array}\right]
$$


It is not dificult to see that $W^{i}(\mathcal{G})=0(i \in\{0,1\})$, that

$$
\begin{aligned}
W^{0}((K)) & =K \\
W^{0}\left(\left(R_{\tau} K_{1} R_{\tau}+R_{\tau} K_{2} V_{-\tau}+V_{\tau} K_{3} R_{\tau}+V_{\tau} K_{4} V_{-\tau}\right)\right) & =0
\end{aligned}
$$

and

$$
\begin{aligned}
W^{1}((K)) & =0 \\
W^{1}\left(\left(R_{\tau} K_{1} R_{\tau}+R_{\tau} K_{2} V_{-\tau}+V_{\tau} K_{3} R_{\tau}+V_{\tau} K_{4} V_{-\tau}\right)\right) & =\left[\begin{array}{ll}
K_{1} & K_{2} \\
K_{3} & K_{4}
\end{array}\right]
\end{aligned}
$$

for every $K, K_{k} \in \mathcal{K}$.

The sets $\left\{j_{0}+\mathcal{G}: j_{0} \in \mathcal{J}_{0}\right\}$ and $\left\{j_{1}+\mathcal{G}: j_{1} \in \mathcal{J}_{1}\right\}$ in $\mathcal{F} / \mathcal{G}$ are naturally closed twosided ideals and so let $\mathcal{J}$ denote the smallest closed two-sided ideal in $\mathcal{F} / \mathcal{G}$ containing both.

We are now in the conditions to apply the lifting theorem [9: Theorem 1.8] and obtain the following result.

Theorem 2.4. The coset $\left(A_{\tau}\right)+\mathcal{G}$ is invertible in the quotient algebra $\mathcal{F} / \mathcal{G}$ if and only if the operators $W^{0}\left(\left(A_{\tau}\right)\right)$ and $W^{1}\left(\left(A_{\tau}\right)\right)$ are invertible in $\mathcal{L}\left(L^{2}\right)$ and $\mathcal{L}\left(L^{2}\right)^{2 \times 2}$, respectively, and the coset $\left(A_{r}\right)+\mathcal{J}$ is invertible in $\mathcal{F}^{\mathcal{J}}:=\mathcal{F} / \mathcal{J}$.

If the sequence $\left(A_{\tau}\right)$ is of the form $\left(P_{\tau} A P_{\tau}+Q_{\tau}\right)$, then $W^{1}\left(A_{\tau}\right)=\left[\begin{array}{cc}A_{11} & 0 \\ 0 & I\end{array}\right]$ and so Theorem 2.4 gives

Corollary 2.5. The method (1) applies to $A$ if and only if $A$ and $A_{11}$ are invertible, and $\left(A_{r}\right)+\mathcal{J}$ is invertible in $\mathcal{F}^{\mathcal{J}}$.

Due to this last result and the fact that in the previous literature the strong limit $A_{11}$ is usually represented by $\tilde{A}$, we will use in the future $\tilde{A}$ instead of $A_{11}$.

Having the above results regarding the algebra $\mathcal{F}$, it must now be verified that this algebra actually contains the sequences that interest us. For that we have the next propositions. Their proofs are either immediate or very similar to one another. We decided to present only the proofs that need some new idea. Also, without reference there will be made extensive use of the properties and relations described in Lemma 1.1 .

Proposition 2.6. The following relations hold:

$$
\begin{array}{rrrr}
R_{\tau} P_{\tau} R_{\tau} \rightarrow I & R_{r} P_{\tau} V_{\tau}=0 & V_{-\tau} P_{\tau} R_{\tau}=0 & V_{-\tau} P_{\tau} V_{\tau}=0 \\
R_{\tau} J R_{\tau} \rightarrow J & R_{\tau} J V_{\tau}=0 & V_{-\tau} J R_{\tau}=0 & V_{-\tau} J V_{\tau}=J
\end{array}
$$

Proof. The proof of these results is immediate

Proposition 2.7. The following relations hold for $c \in P C(\dot{\mathbb{R}})$ :

$$
\begin{aligned}
R_{\tau} c R_{\tau} & \rightarrow c(-\infty) \chi_{-}+c(+\infty) \chi_{+} \\
R_{\tau} c V_{\tau} & =0 \\
V_{-r} c R_{\tau} & =0 \\
V_{-r} c V_{\tau} & \rightarrow c(-\infty) \chi_{-}+c(+\infty) \chi_{+} .
\end{aligned}
$$


Proof. We have

$$
\left(R_{\tau} c R_{\tau} u\right)(x)= \begin{cases}c(\tau-x) u(x) & \text { if } 0<x<\tau \\ c(-\tau-x) u(x) & \text { if }-\tau<x<0 \\ 0 & \text { if }|x|>\tau\end{cases}
$$

For a more simple notation suppose that $\operatorname{supp}(c) \subset \mathbb{R}^{+}$. We must then prove that $\left\|\left(R_{\tau} c R_{r}-c(+\infty) \chi_{+}\right) u\right\| \rightarrow 0$ for any fixed $u \in L^{2}$. Let $m=\max _{x}|c(x)-c(+\infty)|$. For any $\varepsilon>0$ there exists $\tau_{1}$ such that

$$
\tau \geq \tau_{1} \quad \Longrightarrow \quad\left\{\begin{array}{l}
|c(\tau)-c(+\infty)|<\varepsilon \\
\int_{\tau}^{+\infty}|u(x)|^{2} d x<\varepsilon
\end{array}\right.
$$

If $\tau_{0}=2 \tau_{1}$, then we have for $\tau \geq \tau_{0}$

$$
\begin{aligned}
\left\|\left(R_{\tau} c R_{\tau}-c(+\infty) \chi_{+}\right) u\right\|^{2} & \\
= & \int_{0}^{\tau}|(c(\tau-x)-c(+\infty)) u(x)|^{2} d x+\int_{\tau}^{+\infty}|c(+\infty) u(x)|^{2} d x \\
= & \int_{0}^{\tau-\tau_{1}}|(c(\tau-x)-c(+\infty)) u(x)|^{2} d x \\
& +\int_{\tau-\tau_{1}}^{\tau}|(c(\tau-x)-c(+\infty)) u(x)|^{2} d x+\int_{\tau}^{+\infty}|c(+\infty) u(x)|^{2} d x \\
\leq & \varepsilon^{2} \int_{0}^{\tau-\tau_{1}}|u(x)|^{2} d x+\left(m^{2}+c(+\infty)^{2}\right) \varepsilon
\end{aligned}
$$

which ends the proof of the first assertion. The second and third assertions are immediate. For the last one we have only to note that

$$
\left(V_{-\tau} c V_{\tau} u\right)(x)= \begin{cases}c(x+\tau) u(x) & \text { if } x>0 \\ c(x-\tau) u(x) & \text { if } x<0\end{cases}
$$

and to use a reasoning similar to the one above

For a function $a \in P C(\dot{\mathbb{R}})$ let $\tilde{a}(x)=a(-x)$. Define also the operator $R_{\tau}^{\prime}$ as

$$
\left(R_{\tau}^{\prime} u\right)(x)= \begin{cases}u(2 \tau-x) & \text { if } 0<x<\tau \\ u(-2 \tau-x) & \text { if }-\tau<x<0 \\ 0 & \text { if }|x|>\tau .\end{cases}
$$

It is not difficult to see that $\left\|R_{r}^{\prime}\right\|=1$ and $R_{\tau}^{\prime} \rightarrow 0$. 
Proposition 2.8. The following relations hold for $a \in P C(\dot{\mathbb{R}})$ :

$$
\begin{aligned}
& R_{\tau} \chi_{+} \stackrel{\circ}{W}(a) \chi_{+} R_{\tau}=P_{\tau} \chi_{+} \dot{W}(\tilde{a}) \chi_{+} P_{\tau} \\
& R_{\tau} \chi_{-} \stackrel{\circ}{W}(a) \chi_{-} R_{\tau}=P_{r} \chi_{-} \dot{W}(\tilde{a}) \chi_{-} P_{\tau} \\
& R_{\tau} \chi_{+} \stackrel{\circ}{W}(a) \chi_{-} R_{\tau} \rightarrow 0 \\
& R_{\tau} \chi_{-} \stackrel{\circ}{W}(a) \chi_{+} R_{\tau} \rightarrow 0 .
\end{aligned}
$$

Proof. The first two assertions are easily proved by writing the operators explicitly. The third and fourth assertions are similar, and we will only prove the third. If $x<0$ or $x>\tau$, the function $R_{\tau} \chi_{+} \mathscr{W}(a) \chi_{-} R_{\tau} u$ gives the value 0 . For $0<x<\tau$ we have

$$
\begin{aligned}
\left(R_{\tau} \chi_{+} \stackrel{\circ}{W}(a) \chi_{-} R_{\tau} u\right)(x) & =\frac{1}{2 \pi} \int_{-\infty}^{+\infty} e^{-i(\tau-x) \xi} a(\xi) \int_{-\tau}^{0} e^{i \xi y} u(-\tau-y) d y d \xi \\
& =\frac{1}{2 \pi} \int_{-\infty}^{+\infty} e^{-i(-(2 \tau-x))(-\xi)} a(\xi) \int_{-\tau}^{0} e^{i(-\xi) y^{\prime}} u\left(y^{\prime}\right) d y^{\prime} d \xi
\end{aligned}
$$

and this means

$$
R_{\tau} \chi_{+} \stackrel{W}{W}(a) \chi_{-} R_{\tau}=R_{\tau}^{\prime} J \chi_{-} \dot{W}(\tilde{a}) \chi_{-} P_{\tau}
$$

which converges strongly to zero

The proofs of the following propositions are now similar to the above one.

Proposition 2.9. The following relations hold for $a \in P C(\dot{\mathbb{R}})$ :

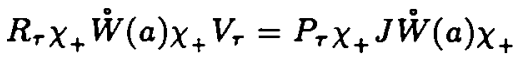

$$
\begin{aligned}
& R_{r} \chi_{-} \stackrel{\mathscr{W}}{(a) \chi_{-}} V_{\tau}=P_{\tau} \chi_{-} J \stackrel{\circ}{W}(a) \chi_{-} \\
& R_{\tau} \chi_{+} \stackrel{\dot{W}}{(a) \chi_{-}} V_{\tau} \rightarrow 0 \\
& R_{\tau} \chi_{-} \stackrel{ }{W}(a) \chi_{+} V_{\tau} \rightarrow 0 \text {. }
\end{aligned}
$$

Proposition 2.10. The following relations hold for $a \in P C(\dot{\mathbb{R}})$ :

$$
\begin{aligned}
& V_{-\tau} \chi_{+} \dot{W}(a) \chi_{+} R_{\tau}=\chi_{+} \stackrel{\circ}{W}(a) J \chi_{+} P_{\tau} \\
& V_{-\tau} \chi_{-} \dot{W}(a) \chi_{-} R_{\tau}=\chi_{-} \dot{W}(a) J \chi_{-} P_{\tau} \\
& V_{-\tau} \chi_{+} \stackrel{\circ}{W}(a) \chi_{-} R_{\tau} \rightarrow 0 \\
& V_{-\tau} \chi_{-} \dot{W}(a) \chi_{+} R_{\tau} \rightarrow 0 .
\end{aligned}
$$

Proposition 2.11. The following relations hold for $a \in P C(\dot{\mathbb{R}})$ :

$$
\begin{aligned}
& V_{-\tau} \chi_{+} \stackrel{\circ}{W}(a) \chi_{+} V_{\tau}=\chi_{+} \stackrel{\circ}{W}(a) \chi_{+} \\
& V_{-\tau} \chi_{-} \stackrel{\circ}{W}(a) \chi_{-} V_{\tau}=\chi_{-} \stackrel{\circ}{W}(a) \chi_{-} \\
& V_{-\tau} \chi_{+} \stackrel{\circ}{W}(a) \chi_{-} V_{\tau} \rightarrow 0 \\
& V_{-\tau} \chi_{-} \stackrel{\circ}{W}(a) \chi_{+} V_{\tau} \rightarrow 0 .
\end{aligned}
$$


Now we are able to define subalgebras of $\mathcal{F}$ that contain the sequences we are interested in. Let $\mathcal{A}$ be the $C^{*}$-subalgebra of $\mathcal{F}$ generated by the constant sequences $(c I)$ and $(\dot{W}(a))$, with $a, c \in P C(\dot{\mathbb{R}})$, and by the sequence $\left(P_{\tau}\right)$. Denote by $\mathcal{A}^{\mathcal{J}}$ the quotient algebra

$$
\frac{\mathcal{A}}{\mathcal{A} \cap \mathcal{J}} \cong \frac{\mathcal{A}+\mathcal{J}}{\mathcal{J}} \text {. }
$$

Let $\mathcal{A}^{\prime}$ be the $C^{*}$-subalgebra of $\mathcal{F}$ generated by the constant sequences $(c I)$ and $(\stackrel{W}{W}(a))$, with $a, c \in P C(\dot{\mathbb{R}})$, by $(J)$, and by the sequence $\left(P_{\tau}\right)$. Denote by $\mathcal{A}^{\prime \mathcal{J}}$ the quotient algebra

$$
\frac{\mathcal{A}^{\prime}}{\mathcal{A}^{\prime} \cap \mathcal{J}} \cong \frac{\mathcal{A}^{\prime}+\mathcal{J}}{\mathcal{J}}
$$

Note that $\mathcal{A}^{\mathcal{J}} \subset \mathcal{A}^{\mathfrak{J}}$.

We will start by studying the simpler algebra $\mathcal{A}^{\mathcal{J}}$. This algebra has a rich center, and we will make use of it through localization in order to obtain invertibility criteria for its elements. It will be used the local principle of Allan, which is a generalization of the Gelfand theory to non-commutative Banach algebras, but that are close to the commutative ones by having a rich center. We will only briefly describe the principle, applied to our $C^{*}$-case. For more detailed information we recommend the reader to [9: Chapter 1]. Let $\mathcal{X}$ be a unital $C^{*}$-algebra and $\mathcal{Y}$ be a closed unital $C^{*}$-subalgebra of the center of $\mathcal{X}$. Then $\mathcal{Y}$ is a commutative algebra and we denote its maximal ideal space by $M(\mathcal{Y})$. To each element $x$ of $M(\mathcal{Y})$ we associate the smallest closed two-sided ideal $I_{x}$ of $\mathcal{X}$ which contains $x$. By $\Phi_{x}$ we denote the canonical homomorphism from $\mathcal{X}$ onto the quotient algebra $\mathcal{X} / I_{x}$. Then Allan's local principle affirms that an element $a \in \mathcal{X}$ is invertible if and only if the cosets $\Phi_{x}(a)$ are invertible in the quotient (=local) algebras $\mathcal{X} / I_{x}$ for every $x \in M(\mathcal{Y})$, and that the mapping $M(\mathcal{Y}) \rightarrow \mathbb{R}^{+}, x \mapsto\left\|\Phi_{x}(a)\right\|$ is upper semi-continuous for each $a \in \mathcal{A}$.

2.4 The algebra with multiplication and convolution operators. As said above, the use of local principles is very important for the understanding of the structure of the algebra $\mathcal{A}^{\mathcal{J}}$.

2.4.1 First localization and identification. We will start by finding a central subalgebra of $\mathcal{A}^{\mathcal{J}}$.

Proposition 2.12. The cosets $(f I)+\mathcal{J}$ with $f \in C(\dot{\mathbb{R}})$ belong to the center of $\mathcal{A}^{\mathcal{J}}$.

Proof. As we have immediatly that $P_{\tau} f I=f P_{\tau}$ and $c f I=f c I$, we are only left with the commutator involving the convolution operators $\dot{W}(a)$. As, e.g. in [17: Proposition 12.6] it can be proved that $f \stackrel{W}{W}(a)-\stackrel{\mathscr{W}}{W}(a) f I$ is a compact operator and the result follows

The result of Proposition 2.12 means that we can apply Allan's local principle (see above or, for instance, [9: Theorem 1.5]) and localize $\mathcal{A}^{\mathcal{J}}$ over the central subalgebra $\mathcal{C}$ generated by the set of cosets $\{(f I)+\mathcal{J}: f \in C(\dot{\mathbb{R}})\}$. The maximal ideal space of this subalgebra is isomorphic to $\dot{\mathbb{R}}$, with a maximal ideal consisting of the cosets $\left\{\left(f_{x} I\right)+\mathcal{J}: f_{x} \in C(\dot{\mathbb{R}}), f_{x}(x)=0\right\}$ (these ideals are indeed not trivial, as one can 
see using the homomorphisms $X_{t}$ defined below). Let $I_{x}$ denote the smallest closed two-sided ideal in $\mathcal{A}^{\mathcal{J}}$ containing the ideal $x$ of $\mathcal{C}$. The result of this localization is that the invertibility problem in $\mathcal{A}^{\mathcal{J}}$ is transferred to an invertibility problem in each of the local algebras $\mathcal{A}_{x}^{\mathcal{J}}:=\mathcal{A}^{\mathcal{J}} / I_{x}$. Let $\Phi_{x}^{\mathcal{J}}$ denote the (canonical) homomorphism from $\mathcal{A}$ to $\mathcal{A}_{\boldsymbol{x}}^{\mathcal{J}} \cdot{ }^{1)}$

Lemma 2.13. If $c \in P C(\dot{\mathbb{R}})$ is continuous at $x$ and $c(x)=0$, then $\Phi_{x}^{J}(c I)=0$.

Proof. For $x \neq \infty$ let $f_{\varepsilon} \in C(\dot{\mathbb{R}})$ such that $0 \leq f_{\varepsilon}<1$ except at $x$, where $f_{\varepsilon}(x)=1$, and let the support of $f_{\varepsilon}$ be contained in the interval $[x-\varepsilon, x+\varepsilon]$. We have that $\Phi_{z}^{\mathcal{J}}\left(f_{\varepsilon} I\right)$ is the identity and then

$$
\left\|\Phi_{x}^{\mathcal{J}}(c I)\right\|=\left\|\Phi_{x}^{\mathcal{J}}(c I) \Phi_{x}^{\mathcal{J}}\left(f_{\varepsilon} I\right)\right\|=\left\|\Phi_{x}^{\mathcal{J}}\left(c f_{\varepsilon} I\right)\right\| \leq\left\|c f_{\varepsilon}\right\|_{L^{\infty}} .
$$

The last norm can be as small as desired by choosing $\varepsilon$ small enough. For $x=\infty$ the proof is similar, with the support of $f_{\varepsilon}$ contained in $\left\{y \in \mathbb{R}:|y|>\frac{1}{\varepsilon}\right\}$

Proposition 2.14. Let $\left(A_{\tau}\right) \in \mathcal{A}$. For $x \neq \infty$ we have $\Phi_{x}^{\mathcal{J}}\left(A_{\tau}\right)=\Phi_{x}^{\mathcal{J}}\left(W^{0}\left(\left(A_{\tau}\right)\right)\right)$.

Proof. The assertion for the constant sequences $\Phi_{x}^{\mathcal{J}}(A)$ is obvious. For $\Phi_{x}^{\mathcal{J}}\left(P_{\tau}\right)$, let $y$ be greater then $|x|$ and define $f_{x}$ to be a continuous function supported in the interval $(-y, y)$ and such that $f_{x}(x)=1$. Then as $\Phi_{x}^{\mathcal{J}}\left(f_{x} I\right)$ is the identity in the local algebra we have

$$
\Phi_{x}^{\mathcal{J}}\left(Q_{\tau}\right)=\Phi_{x}^{\mathcal{J}}\left(f_{x} I\right) \Phi_{x}^{\mathcal{J}}\left(Q_{\tau}\right)=\Phi_{x}^{\mathcal{J}}\left(f_{x} Q_{\tau}\right)
$$

As $\left\|f_{x} Q_{\tau}\right\| \rightarrow 0$, we conclude that $\Phi_{x}^{\mathcal{J}}\left(Q_{\tau}\right)=0$, which means that $\Phi_{x}^{\mathcal{J}}\left(P_{\tau}\right)=\Phi_{x}^{\mathcal{J}}(I-$ $\left.Q_{\tau}\right)=I-\Phi_{x}^{\mathcal{J}}\left(Q_{\tau}\right)=I$

Note that the above result means that the projection $P_{\tau}$ does not appear explicitely in the local algebras, and we can treat local sequences as constant ones. For example, we have

$$
\Phi_{x}^{\mathcal{J}}\left(P_{\tau} A B P_{\tau} C P_{\tau}\right)=\Phi_{x}^{\mathcal{J}}\left(P_{\tau}\right) \Phi_{x}^{\mathcal{J}}(A B) \Phi_{x}^{\mathcal{J}}\left(P_{\tau}\right) \Phi_{x}^{\mathcal{J}}(C) \Phi_{x}^{\mathcal{J}}\left(P_{\tau}\right)=\Phi_{x}^{\mathcal{J}}(A B C)
$$

For a description of the Fredholm properties of the elements of this and other algebras see $[6,17]$. It is possible now to obtain invertibility criteria for all local algebras $\mathcal{A}_{\boldsymbol{x}}^{\mathcal{J}}$ with $x$ finite.

Corollary 2.15. If $W^{0}\left(\left(A_{\tau}\right)\right)$ is a Fredholm operator, then $\Phi_{x}^{\mathcal{J}}\left(A_{\tau}\right)$ is invertible in $\mathcal{A}_{x}^{\mathcal{J}}$ for all $x \in \mathbb{R}$

Proof. If $A=W^{0}\left(\left(A_{\tau}\right)\right)$ is a Fredholm operator, there exist an operator $B$ (with $(B) \in \mathcal{A}$ ) and compact operators $K_{1}$ and $K_{2}$ such that $A B=I+K_{1}$ and $B A=I+K_{2}$. But then $\Phi_{x}^{\mathcal{J}}\left(A_{\tau}\right) \Phi_{x}^{\mathcal{J}}(B)=\Phi_{x}^{\mathcal{J}}\left(I+K_{1}\right)=I$. Also $\Phi_{x}^{\mathcal{J}}(B) \Phi_{x}^{\mathcal{J}}\left(A_{\tau}\right)=I$, and we proved that $\Phi_{x}^{\mathcal{J}}\left(A_{\boldsymbol{r}}\right)$ is invertible

2.4.2 Second localization. In the case $x=\infty$, the algebra $\mathcal{A}_{x}^{\mathcal{J}}$ is still too large for a positive identification. But as this algebra still has a rich center, we can localize again.

1) In order not to burden the notation, for $\left(A_{\tau}\right) \in \mathcal{A}$ we write $\Phi_{x}^{\mathcal{J}}\left(A_{\tau}\right)$ instead of $\Phi_{x}^{\mathcal{J}}\left(\left(A_{\tau}\right)\right)$. 
Proposition 2.16. The cosets $\Phi_{\infty}^{\mathcal{J}}(\dot{W}(f))$ with $f \in C(\dot{\mathbb{R}})$ are in the center of $\mathcal{A}_{\infty}^{\mathcal{J}}$.

Proof. We have that, for any $c \in P C(\dot{\mathbb{R}}), \stackrel{W}{W}(f) c I-c \dot{W}(f)$ is compact by, e.g. [6] or [17: Proposition 12.6]. We will prove now that the commutator $W(f) P_{\tau}-P_{\tau} W(f)$ is in $\mathcal{J}$. Write

$$
\stackrel{\circ}{W}(f) P_{\tau}-P_{\tau} \dot{W}(f)=Q_{\tau} \stackrel{ }{W}(f) P_{\tau}-P_{\tau} \stackrel{\circ}{W}(f) Q_{\tau} .
$$

As $Q_{r} \rightarrow 0$ and the operators $\chi_{ \pm} \stackrel{\circ}{W}(f) \chi_{\mp}$ are compact, we have that $Q_{\tau} \chi_{ \pm} \stackrel{\circ}{W}(f) \chi_{\mp}$ and $\chi_{ \pm} \dot{W}(f) \chi_{\mp} Q_{\tau}$ converge to zero uniformly, and so the corresponding sequences belong to $\mathcal{G}$. We are only left with the sequences $\left(Q_{\tau} \chi_{ \pm} \stackrel{\circ}{W}(f) \chi_{ \pm} P_{\tau}\right)$ and $\left(P_{\tau} \chi_{ \pm} \stackrel{\circ}{W}(f) \chi_{ \pm} Q_{\tau}\right)$. For them we can write

$$
Q_{\tau} \chi_{ \pm} \dot{W}(f) \chi_{ \pm} P_{\tau}=V_{\tau} V_{-\tau} \chi_{ \pm} \dot{W}(f) \chi_{ \pm} R_{\tau} R_{\tau}
$$

and by the use of Proposition 2.10 these last operators are equal to $V_{\tau} \chi_{ \pm} \stackrel{W}{W}(f) J_{\chi \pm} P_{\tau} R_{\tau}$ with $\chi_{ \pm} W(f) J_{ \pm}$compact, and so the sequences are in $\mathcal{J}_{1}$. Equally, the sequences $\left(P_{\tau} \chi_{ \pm} W(f) \chi_{ \pm} Q_{\tau}\right)$ by the use of Proposition 2.9 can be seen to be in $\mathcal{J}_{1}$, which finishes the proof

This last result means that we can localize $\mathcal{A}_{\infty}^{\mathcal{J}}$ over the maximal ideal space of the subalgebra $\mathcal{C}^{\prime}$ generated by the cosets $\Phi_{\infty}^{\mathcal{J}}(\stackrel{W}{W}(f))$ with $f \in C(\dot{\mathbb{R}})$. This maximal ideal space is formed by the cosets $\Phi_{\infty}^{\mathcal{J}}\left(\dot{W}\left(f_{x}\right)\right)$ with $f_{x}(x)=0$ and $x \in \dot{\mathbb{R}}$, and it is isomorphic to $\dot{\mathbb{R}}$ (that these maximal ideals are indeed not trivial can be verified by the homomorphisms $W_{\text {s }}$ defined below).

In order to apply the local principle of Allan, let $I_{\infty, x}(x \in \dot{\mathbb{R}})$ be the smallest closed two-sided ideal of $\mathcal{A}_{\infty}^{\mathcal{J}}$ which contains the ideal $x$ of $\mathcal{C}^{\prime}$. We call $\Phi_{\infty, x}^{\mathcal{J}}$ the homomorphism which is the composition of the canonical homomorphism from $\mathcal{A}_{\infty}^{\mathcal{J}}$ to $\mathcal{A}_{\infty, x}^{\mathcal{J}}:=\mathcal{A}_{\infty}^{\mathcal{J}} / I_{\infty, x}$ with $\Phi_{\infty}^{J}$. The following lemma corresponds to Lemma 2.13. 0 .

Lemma 2.17. If $a \in P C(\dot{\mathbb{R}})$ is continuous at $x$ and $a(x)=0$, then $\Phi_{\infty, x}^{\mathcal{J}}\left(\dot{W}^{\prime}(a)\right)=$

Proof. For $x \neq \infty$ let $f_{\varepsilon} \in C(\dot{\mathbb{R}})$ be such that $0 \leq f_{\varepsilon}<1$ except at $x$, where $f_{e}(x)=1$, and let the support of $f_{\varepsilon}$ be contained in the interval $[x-\varepsilon, x+\varepsilon]$. We have that $\Phi_{\infty, x}^{\mathcal{J}}\left(\mathscr{W}\left(f_{e}\right)\right)$ is the identity and then

$$
\left\|\Phi_{\infty, x}^{\mathcal{J}}(\stackrel{\circ}{W}(a))\right\|=\left\|\Phi_{\infty, x}^{\mathcal{J}}(\stackrel{\circ}{W}(a)) \Phi_{\infty, x}^{\mathcal{J}}\left(\stackrel{\circ}{W}\left(f_{e}\right)\right)\right\|=\left\|\Phi_{\infty, x}^{\mathcal{J}}\left(\stackrel{\circ}{W}\left(a f_{e}\right)\right)\right\| \leq\left\|a f_{e}\right\|_{L^{\infty}}
$$

The last norm can be as small as desired by choosing $\varepsilon$ small enough. For $x=\infty$ the proof is the same, but the support of $f_{\varepsilon}$ is contained in $\left\{y \in \dot{\mathbb{R}}:|y|>\frac{1}{\varepsilon}\right\}$

2.4.3 Identification of the local algebras - the needed homomorphisms. Our objective now is to obtain enough information about the local algebras $\mathcal{A}_{\infty, x}^{\mathcal{J}}$ so that invertibility criteria can be derived for their elements. For this we will construct symbol 
homomorphisms that give us locally equivalent representations (see [9]). For our objective of constructing symbol homomorphisms for the local algebras, we need to use the following operators, defined in $L^{2}(\mathbb{R})$ :

$$
\begin{aligned}
& \left(Z_{\tau} u\right)(x)=u\left(\frac{x}{\tau}\right) \quad \text { with } Z_{\tau}^{-1}=Z_{\tau-1} \text { and }\left\|Z_{\tau}\right\|=\sqrt{\tau}(\tau>0) \\
& \left(U_{s} u\right)(x)=e^{-i x s} u(x) \quad \text { with } U_{s}^{-1}=U_{-s} \text { and }\left\|U_{s}\right\|=1 \quad(s \in \mathbb{R}) .
\end{aligned}
$$

Given now a sequence $\left(A_{\tau}\right) \in \mathcal{A}$, we can define a transformation $W_{\text {sr }}$ such that to the operator $A_{\tau}$ associates the operator

$$
W_{s+}\left(A_{\tau}\right)=Z_{\tau}^{-1} U_{-s} A_{\tau} U_{s} Z_{\tau} \quad(s \in \mathbb{R}) .
$$

Note that $W_{s r}$ is multiplicative, i.e. $W_{s \tau}\left(A_{\tau} B_{\tau}\right)=W_{s \tau}\left(A_{\tau}\right) W_{s \tau}\left(B_{\tau}\right)$, and

$$
\left\|W_{s \tau}\left(A_{\tau}\right)\right\|_{\mathcal{L}\left(L^{2}\right)} \leq\left\|A_{\tau}\right\|_{\mathcal{L}\left(L^{2}\right)} .
$$

Defining now if it exists the strong limit

$$
W_{s}\left(A_{\tau}\right)=\mathrm{s}-\lim _{\tau \rightarrow \infty} W_{s \tau}\left(A_{\tau}\right),
$$

we obtain the next lemma, where $a\left(x^{ \pm}\right)=\lim _{y \rightarrow x \pm} a(y)$.

Lemma 2.18. If $\left(A_{\tau}\right) \in \mathcal{A}$, then the limit $W_{s}\left(A_{\tau}\right)$ exists. In particular:

a) $W_{s}\left(P_{\tau}\right)=P_{1}$.

b) $W_{s}(c I)=c(-\infty) \chi_{-}+c(+\infty) \chi_{+}$for $c \in P C(\dot{\mathbb{R}})$.

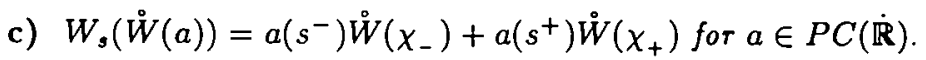

d) If $\left(j_{\tau}\right) \in \mathcal{J}_{0}$ or $\left(j_{\tau}\right) \in \mathcal{J}_{1}$, then $W_{s}\left(j_{\tau}\right)=0$.

Proof. Assertion a) can be proved by a simple calculation. Assertions b) and c) are proved in [17: Proposition 13.1]. To prove the last one note that, for $G_{\tau} \in \mathcal{G}$, $\left\|W_{s \tau}\left(G_{\tau}\right)\right\| \leq\left\|G_{\tau}\right\| \rightarrow 0$. Also, $W_{s}(K)=0$ for $K \in \mathcal{K}$, because $\frac{1}{\sqrt{\tau}} Z_{\tau}$ tends weakly to zero, $U_{-s} K U_{s}$ is compact and $\sqrt{\tau} Z_{\tau}^{-1}$ is uniformly bounded. Writing $W_{s r}\left(R_{r}\right)$ and $W_{s r}\left(V_{-\tau}\right)$ explicitely we obtain $W_{s \tau}\left(R_{\tau}\right)=e^{i s(2 \tau x-\operatorname{sgn}(x) \tau)} R_{1}$ and $W_{s \tau}\left(V_{-\tau}\right)=$ $e^{-i s \operatorname{sgn}(x) r} V_{-1}$, which have no strong limit. But the fact that $\frac{1}{\sqrt{\tau}} R_{\tau} U_{s} Z_{\tau}, \frac{1}{\sqrt{\tau}} V_{-\tau} U_{s} Z_{\tau}$ tend weakly to zero and that $\sqrt{\tau} Z_{\tau}^{-1} U_{-s} R_{\tau}$ and $\sqrt{\tau} Z_{\tau}^{-1} U_{-s} V_{\tau}$ are uniformly bounded permit us again to conclude the assertion

Define also the unitary operator $\tilde{V}_{\mathrm{T}}$ acting on $L^{2}$ by

$$
\left(\tilde{V}_{r} u\right)(x)=u(x-\tau), \quad \text { with } \tilde{V}_{\tau}^{-1}=\tilde{V}_{-\tau} \text { and }\left\|\tilde{V}_{r}\right\|=1 \quad(\tau \in \mathbb{R}) .
$$

With this operator, in a similar way to the one used above, we can define the transformations $W_{\infty}^{ \pm}$that associate to an operator $A_{\tau}$ the operator

$$
W_{\infty \tau}^{ \pm}\left(A_{\tau}\right)=\tilde{V}_{\mp \tau} A_{\tau} \tilde{V}_{ \pm \tau}
$$

and the strong limit

$$
W_{\infty}^{ \pm}\left(A_{r}\right)=\mathrm{s}-\lim _{r \rightarrow \infty} W_{\infty r}^{ \pm}\left(A_{\tau}\right)
$$

if it exists. We would like now to prove a result similar to Lemma 2.18. But for this we must first prove a very simple auxiliary result from general operator theory in Hilbert spaces. 
Lemma 2.19. Let $\left(A_{\tau}\right)$ be a sequence that tends weakly to an operator $A$, such that $\left(A_{\tau}^{*}\right)$ tends strongly to $A^{*}$. Then if $\left(B_{\tau}\right)$ is a sequence that tends strongly to an operator $B$, we have that $\left(A_{\tau} B\right)$ and $\left(A_{\tau} B_{\tau}\right)$ tend weakly to $A B$.

Proof. If $(\cdot, \cdot)$ denotes the duality product, we have for any $u, v \in L^{2}$

$$
\begin{aligned}
\left(v, A_{\tau} B u\right) & \rightarrow(v, A B u) \\
\left(v, A_{\tau} B_{\tau} u\right)=\left(A_{\tau}^{*} v, B_{\tau} u\right) & \rightarrow\left(A^{*} v, B u\right)=(v, A B u)
\end{aligned}
$$

and the assertion is proved

Now we are in the conditions to prove

Lemma 2.20. The strong limits $W_{\infty}^{ \pm}\left(A_{\tau}\right)$ exist for all $\left(A_{\tau}\right) \in \mathcal{A}$. In particular,

a) $W_{\infty}^{ \pm}\left(P_{\tau}\right)=\chi_{\mp}$ and $W_{\infty}^{ \pm}\left(Q_{\tau}\right)=\chi_{ \pm}$.

b) $W_{\infty}^{ \pm}(c I)=c( \pm \infty) I$.

c) $W_{\infty}^{ \pm}(\stackrel{\circ}{W}(a))=\stackrel{\circ}{W}(a)$.

d) If $\left(j_{r}\right) \in \mathcal{A} \cap \mathcal{J}$, then $W_{\infty}^{ \pm}\left(j_{\tau}\right) \in \mathcal{K}$.

Proof. Assertion a) can be proved by a simple calculation. To prove assertion b) note that $\left(W_{\infty r}^{ \pm}(c I) u\right)(x)=c(x \pm \tau) u(x)$, and assertion c) follows from $W_{\infty r}^{ \pm}\left(\mathscr{W}^{\circ}(a)\right)=$ $\stackrel{\circ}{W}(a)$. To prove the last assertion note again that, for $G_{\tau} \in \mathcal{G},\left\|W_{\infty \tau}^{ \pm}\left(G_{\tau}\right)\right\| \leq\left\|G_{\tau}\right\| \rightarrow 0$. For $\left(j_{\tau}\right) \in \mathcal{J}_{0}$, note that $\tilde{V}_{\tau}$ and $\tilde{V}_{-\tau}$ tend weakly to zero as $\tau \rightarrow \infty$. In relation to $\left(j_{\tau}\right) \in \mathcal{J}_{1}$, we have the strong limits $R_{\tau} \tilde{V}_{ \pm \tau} \rightarrow \chi_{ \pm} J, V_{-\tau} \tilde{V}_{ \pm r} \rightarrow \chi_{ \pm}$and the weak limits $\tilde{V}_{\mp \tau} R_{\tau} \rightarrow J \chi_{ \pm}, \tilde{V}_{\mp} V_{\tau} \rightarrow \chi_{ \pm}$when $\tau_{.} \rightarrow+\infty$. By Lemma 2.19 this means that $W_{\infty r}^{ \pm}\left(j_{r}\right)$ tends weakly to a compact operator $K \in \mathcal{K}$. But as $\left(j_{r}\right)$ also belongs to $\mathcal{A}$ and for this algebra by assertions a) - c) the strong limit is well defined, we conclude that $W_{\infty r}^{ \pm}\left(j_{r}\right)$ tends strongly to $K$

We are now in the position to identify the local algebras.

2.4.4 The local algebra $\mathcal{A}_{\infty, s}^{\mathcal{J}}(s \in \mathbb{R})$. By Lemma 2.18 the strong limit $W_{s}$ is an algebra homomorphism between the algebras $\mathcal{A}$ and $\mathcal{B}:=\operatorname{alg}\left(I, P_{1}, \chi_{+}, \dot{W}\left(\chi_{+}\right)\right)$. Define now the application

$$
W_{s}^{\prime}: \mathcal{B} \rightarrow \mathcal{A}_{\infty, s}^{\mathcal{J}}, \quad W_{s}^{\prime}(A)=\Phi_{\infty, s}\left(U_{s} Z_{\tau} A Z_{\tau}^{-1} U_{-s}\right)
$$

This application is also an algebra homomorphism (note that $\left(U_{s} Z_{\tau} A Z_{T}^{-1} U_{-s}\right)_{r \in \mathbb{R}^{+}} \in \mathcal{A}$ for any $A \in \mathcal{B}$ as one can see by the generators of $\mathcal{B}$ ), and so the following result holds.

Proposition 2.21. The local algebre $\mathcal{A}_{\infty, \mathrm{J}}^{\mathcal{J}}$ is isomorphic to the algebra $\mathcal{B}$, and the isomorphism is given by $W_{\mathrm{s}}$.

Proof. We must first see that $W_{s}$ is well defined in $\mathcal{A}_{\infty, s}^{\mathcal{J}}$. Having already the results from Lemma 2.18 , we have only to prove that $W_{s}\left(A_{r}\right)=0$ for the sequences $\left(A_{\tau}\right)$ in the ideal $I_{\infty, s}$. This follows also from Lemma $2.18 / \mathrm{c}$ ), where we can see that 
$W_{s}(\stackrel{W}{W}(a))=0$ for $a \in C(\dot{\mathbb{R}})$ such that $a(s)=0$. And so it makes sense to define the quotient homomorphism

$$
W_{s}: \mathcal{A}_{\infty, s}^{\mathcal{J}} \rightarrow \mathcal{B}
$$

which for simplicity is represented by the same symbol.

We are left with proving that the homomorphism $W_{s}$ is really an isomorphism. If $\Phi_{\infty, s}^{J}\left(A_{\tau}\right)$ is invertible, it is easy to see that $W_{s}\left(\Phi_{\infty, s}^{J}\left(A_{r}\right)\right)$ is also invertible, because $W_{s}$ is a unital homomorphism. For the reverse direction we have that $W_{s}^{\prime}$ is also a unital homomorphism, and if we can prove that

$$
W_{s}^{\prime}\left(W_{s}\left(\Phi_{\infty, s}^{\mathcal{J}}\left(A_{\tau}\right)\right)=\Phi_{\infty, s}^{\mathcal{J}}\left(A_{r}\right)\right.
$$

our proof is finished. As $W_{s}^{\prime}, W_{s}$ and $\Phi_{\infty, \text {, }}^{\mathcal{J}}$ are all homomorphisms, it is sufficient to prove (35) for the cosets generating the algebra. But for these cosets the result is a direct consequence of the homomorphisms definitions and Lemmas 2.13 and 2.17

2.4.5 The local algebra $\mathcal{A}_{\infty, \infty}^{\mathcal{J}}$. By the use of Lemmas 2.13 and 2.17 it is easy to see that this algebra is generated by the three projections $\Phi_{\infty, \infty}^{\mathcal{J}}\left(\chi_{+}\right), \Phi_{\infty, \infty}^{\mathcal{J}}\left(\dot{W}^{\circ}\left(\chi_{+}\right)\right)$and $\Phi_{\infty, \infty}^{\mathcal{J}}\left(P_{\tau}\right)$. This algebra still has a non-trivial center, as we can see next.

Proposition 2.22. The projection $\Phi_{\infty, \infty}^{\mathcal{J}}\left(\chi_{+}\right)$belongs to the center of $\mathcal{A}_{\infty, \infty}^{\mathcal{J}}$.

Proof. As $P_{r}$ commutes with $\chi_{+}$, the only relation that needs to be proved, is

$$
\Phi_{\infty, \infty}^{\mathcal{J}}\left(\stackrel{\mathscr{W}}{ }\left(\chi_{+}\right) \chi_{+}\right)=\Phi_{\infty, \infty}^{\mathcal{J}}\left(\chi_{+} \stackrel{\circ}{W}\left(\chi_{+}\right)\right) .
$$

For this consider the function $\chi_{+}^{\prime}$, continuous, and taking the value 0 at $-\infty$ and 1 at $+\infty$. By Lemmas 2.13 and 2.17 ,

$$
\Phi_{\infty, \infty}^{\mathcal{J}}\left(\chi_{+}\right)=\Phi_{\infty, \infty}^{\mathcal{J}}\left(\chi_{+}^{\prime}\right) \quad \text { and } \quad \Phi_{\infty, \infty}^{\mathcal{J}}\left(\mathscr{W}^{\prime}\left(\chi_{+}\right)\right)=\Phi_{\infty, \infty}^{\mathcal{J}}\left(\mathscr{W}^{\prime}\left(\chi_{+}^{\prime}\right)\right) \text {. }
$$

This together with the fact that ${ }^{\circ}\left(\chi_{+}^{\prime}\right) \chi_{+}^{\prime}=\chi_{+}^{\prime} \stackrel{\circ}{W}\left(\chi_{+}^{\prime}\right)+K$ for some compact operator $K$ (see [6: Proposition 1.3] or [17: Proposition 12.6/b),(iii)]) ends the proof

Localizing over the maximal ideal space of the central subalgebra generated by the identity and $\Phi_{\infty, \infty}^{\mathcal{J}}\left(\chi_{+}\right)$, let $I_{\infty, \infty \pm}$ denote the smallest closed two-sided ideal in $\mathcal{A}_{\infty, \infty}^{\mathcal{J}}$ containing the maximal ideal (of the central subalgebra) $\mathbb{C} \Phi_{\infty, \infty}^{\mathcal{J}}\left(\chi_{\mp}\right.$ ), and put $\mathcal{A}_{\infty, \infty \pm}^{\mathcal{J}, \infty}=\mathcal{A}_{\infty, \infty}^{\mathcal{J}} / I_{\infty, \infty \pm}$ with $\Phi_{\infty, \infty \pm}^{\mathcal{J}}$ the canonical homomorphism from $\mathcal{A}$ into $\mathcal{A}_{\infty, \infty \pm}^{\mathcal{J}}$. These two local algebras are generated each by two idempotents ${ }^{2)}$, and so we can apply the two projections theorem (see, for instance, [9: Theorem 1.10]). This theorem gives us symbol mappings $N^{ \pm}$to a space of matrix functions:

$$
\begin{aligned}
N^{ \pm}\left(\Phi_{\infty, \infty \pm}^{\mathcal{J}}\left(\chi_{ \pm}\right)\right)=e^{\prime}: & x \mapsto\left[\begin{array}{ll}
1 & 0 \\
0 & 1
\end{array}\right] \\
N^{ \pm}\left(\Phi_{\infty, \infty \pm}^{\mathcal{J}}\left(P_{\tau}\right)\right)=p_{1}^{\prime}: & x \mapsto\left[\begin{array}{ll}
1 & 0 \\
0 & 0
\end{array}\right] \\
N^{ \pm}\left(\Phi_{\infty, \infty \pm}^{\mathcal{J}}\left(\stackrel{\circ}{W}\left(\chi_{+}\right)\right)=p_{2}^{\prime}:\right. & x \mapsto\left[\begin{array}{cc}
x & \sqrt{x(1-x)} \\
\sqrt{x(1-x)} & -1-x
\end{array}\right]
\end{aligned}
$$

2) For example, $\mathcal{A}_{\infty, \infty+}^{J}$ is generated by $\Phi_{\infty, \infty+}^{\mathcal{J}}\left(P_{r}\right)$ and $\Phi_{\infty, \infty+}^{J}\left(\dot{W}\left(\chi_{+}\right)\right)$. 
with

$$
x \in \sigma_{\mathcal{A}_{\infty, \infty \pm}^{\mathcal{J}}}\left(\Phi_{\infty, \infty \pm}^{\mathcal{J}}\left(P_{\tau} \dot{W}\left(\chi_{+}\right) P_{\tau}\right)\right)
$$

if only

$$
\{0,1\} \subset \sigma_{\mathcal{A}_{\infty, \infty \pm}^{\mathcal{J}}}\left(\Phi_{\infty, \infty \pm}^{\mathcal{J}}\left(P_{\tau} \stackrel{\circ}{W}\left(\chi_{+}\right) P_{\tau}\right)\right)
$$

and these are not isolated points of the spectrum. So we are left with finding these local spectra.

To find the local spectra, we will use the strong limits $W_{\infty}^{ \pm}$, which will be seen to be well defined homomorphisms acting on the local algebras $\mathcal{A}_{\infty, \infty \pm}^{\mathcal{J}}$. We start by remarking that, by Lemma $2.20, W_{\infty}^{ \pm}(\mathcal{A} \cap \mathcal{J}) \subset \mathcal{K}$ and $W_{\infty}^{ \pm}\left(I_{\infty}\right)=0$. Then $W_{\infty}^{ \pm}$are well defined from $\mathcal{A}_{\infty}^{\mathcal{J}}$ into the Calkin algebra $\mathcal{N}^{\pi}$, with $\mathcal{N}$ generated by the convolution operators $\dot{W}(a)$ with $a \in P C(\dot{\mathbb{R}})$ and the operator of multiplication by $\chi_{+}$. If $f \in C(\dot{\mathbb{R}})$, then $\dot{W}(f)$ is in the center of this algebra, and we can localize via the local principle of Allan. The maximal ideal space of this central subalgebra is isomorphic to $\dot{\mathbb{R}}$, and to a point $x$ of $\dot{\mathbb{R}}$ it corresponds the maximal ideal of all operators $\dot{W}(f)$ with $f(x)=0$. Let $\mathcal{I}_{x}$ denote the smallest closed two-sided ideal in $\mathcal{N}^{\pi}$ containing the ideal $x$. The set $W_{\infty}^{ \pm}\left(I_{\infty, \infty}\right)$ is then included into $\mathcal{I}_{\infty}$, and so the homomorphisms $W_{\infty}^{ \pm}$are well defined from the local algebra $\mathcal{A}_{\infty, \infty}^{\mathcal{J}}$ into $\mathcal{N}_{\infty}^{\pi}:=\mathcal{N}^{\pi} / \mathcal{I}_{\infty}$. Finally, as $W_{\infty}^{ \pm}\left(I_{\infty, \infty \pm}\right)=0, W_{\infty}^{ \pm}$is well defined in the algebra $\mathcal{A}_{\infty, \infty \pm}^{\mathcal{J}}$, respectively.

The above result means that, for any element $A_{\tau}$ in $\mathcal{A}$,

$$
\sigma_{\mathcal{A}_{\infty, \infty \pm}^{\mathcal{J}}}\left(\Phi_{\infty, \infty \pm}^{\mathcal{J}}\left(A_{\tau}\right)\right) \supset \sigma_{\mathcal{N}_{\infty}^{ \pm}}\left(W_{\infty}^{ \pm}\left(A_{\tau}\right)\right)
$$

Applying this to our particular case we obtain, noting that $\left.W_{\infty}^{ \pm}\left(P_{\tau} \stackrel{\circ}{W}\left(\chi_{+}\right) P_{\tau}\right)\right)=$

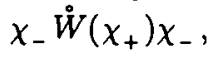

$$
[0,1] \subset \sigma_{\mathcal{A}_{\infty, \infty \pm}^{\mathcal{J}}}\left(\Phi_{\infty, \infty \pm}^{\mathcal{J}}\left(P_{\tau} \dot{W}\left(\chi_{+}\right) P_{\tau}\right)\right)
$$

To prove that the inclusion is really an equality, it is only necessary to remark that

$$
\left\|\Phi_{\infty, \infty \pm}^{\mathcal{J}}\left(P_{\tau} \stackrel{\circ}{W}\left(\chi_{+}\right) P_{\tau}\right)\right\| \leq\left\|P_{\tau} \stackrel{\circ}{W}\left(\chi_{+}\right) P_{\tau}\right\| \leq 1
$$

(which means that the points of the spectrum must have module less or cqual to 1) and that the element $\Phi_{\infty \infty_{ \pm}}^{\mathcal{J}}\left(P_{\tau} \dot{W}\left(\chi_{+}\right) P_{\tau}\right)$ is the image of the positive sequence $\left(P_{r} W\left(\chi_{+}\right) P_{r}\right)$ (which means that the spectrum is contained in the positive half-axis). So define the homomorphism

$$
M^{ \pm}=N^{ \pm} \Phi_{\infty, \infty \pm}^{\mathcal{J}}
$$

We just proved the following result.

Proposition 2.23. The local algebras $\mathcal{A}_{\infty, \infty \pm}^{\mathcal{J}}$ are isomorphic to the unital $C^{*}$. subalgebra of the algebra of $(2 \times 2)$-matrix functions defined on $[0,1]$, which are diagonal at $\{0,1\}$ (and which is generated by $e^{\prime}, p_{1}^{\prime}$ and $p_{2}^{\prime}$ ), and these isomorphisms are given 
by $N^{ \pm}$. The coset $\Phi_{\infty, \infty \pm}^{\mathcal{J}}\left(A_{r}\right)$ is invertible in the local algebra $\mathcal{A}_{\infty, \infty \pm}^{\mathcal{J}}$ if and only if $M^{ \pm}\left(A_{\tau}\right)$ are invertible. In particular,

$$
\begin{aligned}
M^{ \pm}(c I): & x \mapsto\left[\begin{array}{cc}
c( \pm \infty) & 0 \\
0 & c( \pm \infty)
\end{array}\right] \\
M^{ \pm}\left(P_{\tau}\right): & x \mapsto\left[\begin{array}{ll}
1 & 0 \\
0 & 0
\end{array}\right] \\
M^{ \pm}(\stackrel{\circ}{W}(a)): & x \mapsto a(-\infty)\left[\begin{array}{cc}
1-x & -\sqrt{x(1-x)} \\
-\sqrt{x(1-x)} & x
\end{array}\right] \\
& +a(+\infty)\left[\begin{array}{cc}
x & \sqrt{x(1-x)} \\
\sqrt{x(1-x)} & 1-x
\end{array}\right] .
\end{aligned}
$$

So we have solved the problem of identification of the local algebras for the algebra $\mathcal{A}$, and now we can turn our attention to the larger algebra that contains the flip, i.e. the algebra $\mathcal{A}^{\prime}$.

2.5 The algebra with a flip, multiplication and convolution operators. It will be again necessary to use localization principles in order to obtain invertibility criteria for the cosets in $\mathcal{A}^{\prime} \mathcal{J}$.

2.5.1 First localization and identification. This first part is very similar to the case of the algebra without the flip. Let $\tilde{C}(\dot{\mathbb{R}})$ represent the subspace of $C(\dot{\mathbb{R}})$ constituted by the even functions.

Proposition 2.24. The cosets $(f I)+\mathcal{J}$ with $f \in \tilde{C}(\dot{\mathbb{R}})$ belong to the center of $\mathcal{A}^{\prime} \mathcal{J}$.

Proof. As we have that $P_{r} f I=f P_{r}, c f I=f c I$ and $f J=J f I$, the only thing that is left are the convolution operators $W(a)$. As [17: Proposition 12.6] shows that $f \stackrel{W}{W}(a)-\stackrel{W}{W}(a) f I$ is a compact operator, the result follows

So we can also apply here the local principle of Allan, and localize $\mathcal{A}^{\prime} \mathcal{J}$ over the central subalgebra $\mathcal{C}$ generated by the set of cosets $\{(f I)+\mathcal{J}: f \in \tilde{C}(\dot{\mathbb{R}})\}$. The maximal ideal space of this subalgebra is isomorphic to $[0, \infty]$, with a maximal ideal consisting of the cosets $\left\{\left(f_{x} I\right)+\mathcal{J}: f_{x} \in \tilde{C}(\mathbb{R})\right.$ and $\left.f( \pm x)=0\right\}$. Let $I_{x}^{\prime}$ denote the smallest closed two-sided ideal in $\mathcal{A}^{\prime \mathcal{J}}$ containing the ideal $x$ of $\mathcal{C}$. Define the local algebras $\mathcal{A}_{x}^{\prime \mathcal{J}}=\mathcal{A}^{\prime \mathcal{J}} / I_{x}^{\prime}$ and let $\Phi_{x}^{\mathcal{J}}$ (instead of $\Phi^{\prime}{ }_{x}^{\mathcal{J}}$, for simplicity) denote the (canonical) homomorphism from $\mathcal{A}^{\prime}$ to $\mathcal{A}_{\boldsymbol{x}}^{\prime \mathcal{J}}$.

We can now obtain Lemma 2.13 (with the modification that the function must be continuous and take the value 0 at the points $\{-x, x\})$, Proposition 2.14 and Corollary 2.15 in a very similar way to the case without the flip, so we will not repeat the arguments. These three results give the invertibility conditions we need for all the local algebras except the local algebra at infinity. But for the sake of completeness, we proceed here with the identification. For this purpose we will use the homomorphism

$$
X_{\imath}\left(A_{\tau}\right)=\lim _{\tau \rightarrow \infty} Z_{\tau} \tilde{V}_{-t} W^{0}\left(\left(A_{\tau}\right)\right) \tilde{V}_{t} Z_{\tau}^{-1}
$$


which is well defined in the algebra $\mathcal{A}^{\mathcal{J}}$ (without the flip), because $W^{0}\left(\mathcal{J}_{1}\right)=\{0\}$ and $W^{0}\left(\mathcal{J}_{0}\right) \subset \mathcal{K}$ with $\lim _{\tau \rightarrow \infty} Z_{\tau} \tilde{V}_{-t} K \tilde{V}_{t} Z_{\tau}^{-1}=0$ for all $K \in \mathcal{K}$, because $\tilde{V}_{-t} K \tilde{V}_{t}$ is compact, $\frac{1}{\sqrt{\tau}} Z_{\tau}$ is uniformly bounded and $\sqrt{\tau} Z_{\tau}^{-1}$ tends weakly to zero. In the case $t=0, X_{t}$ is even defined in $\mathcal{A}^{\prime} \mathcal{J}$ which, together with the next proposition, will permit us to identify $\mathcal{A}_{0}^{\prime \mathcal{J}}$.

Proposition 2.25. For any $t \in \mathbb{R}_{0}^{+}$and $a \in P C(\dot{\mathbb{R}})$, we have

$$
\Phi_{t}^{\mathcal{J}}(\stackrel{\circ}{W}(a))=\Phi_{t}^{\mathcal{J}}\left(a(-\infty) \stackrel{\circ}{W}\left(\chi_{-}\right)+a(+\infty) \stackrel{\circ}{W}\left(\chi_{+}\right)\right) .
$$

Proof. Let $f \in \tilde{C}(\dot{\mathbb{R}})$ such that $f( \pm t)=1$ and $f( \pm \infty)=0$. Then $\Phi_{t}^{\mathcal{J}}(f I)$ is the identity in the local algebra and we can write putting $a^{\prime}=a(-\infty) \chi_{-}+a(+\infty) \chi_{+}$

$$
\Phi_{\imath}^{\mathcal{J}}(\stackrel{\circ}{W}(a))=\Phi_{t}^{\mathcal{J}}(f \stackrel{\circ}{W}(a))=\Phi_{t}^{\mathcal{J}}\left(f \stackrel{\circ}{W}\left(a^{\prime}\right)\right)+\Phi_{t}^{\mathcal{J}}\left(f \stackrel{\circ}{W}\left(a-a^{\prime}\right)\right)
$$

As $f( \pm \infty)=\left(a-a^{\prime}\right)( \pm \infty)=0, f \stackrel{W}{W}\left(a-a^{\prime}\right)$ is compact (see [6: Proposition 1.2] or [17: Proposition 12.6]) and the result follows

2.5.2 The local algebra $\mathcal{A}_{0}^{\prime \mathcal{J}}$. Now it is possible to identify the local algebra $\mathcal{A}_{0}^{\prime \mathcal{J}}$.

Proposition 2.26. The homomorphism $X_{0}$ is well defined in $\mathcal{A}_{0}^{\prime J}$. Moreover, the local algebra $\mathcal{A}_{0}^{\prime J}$ is isomorphic to the closed algebra $\operatorname{alg}\left(I, \chi_{+}, \stackrel{\circ}{W}\left(\chi_{+}\right), J\right)$, and the isomorphism is given by $X_{0}$. In particular,

$$
\begin{aligned}
X_{0}(\stackrel{\circ}{W}(a)) & =a(-\infty) \stackrel{\circ}{W}\left(\chi_{-}\right)+a(+\infty) \stackrel{\circ}{W}\left(\chi_{+}\right) \\
X_{0}(c I) & =c\left(0^{-}\right) \chi_{-}+c\left(0^{+}\right) \chi_{+}, X_{0}(J)=J, X_{0}\left(P_{\tau}\right)=I .
\end{aligned}
$$

Proof. Regarding the particular values of $X_{0}$ for the generators of $\mathcal{A}_{0}^{\prime \mathcal{J}}$, it is only necessary to remember the proposition correspondent to Proposition 2.14 (for $\Phi_{0}^{\mathcal{J}}\left(P_{\tau}\right)$ ) and to remark that $\left(Z_{\tau} c Z_{\tau}^{-1}\right)(x)=c\left(\frac{x}{\tau}\right), Z_{\tau} \dot{W}(a) Z_{\tau}^{-1}=\dot{W}\left(a_{\tau}\right)$ with $a_{\tau}(\xi)=a(\xi \tau)$, and $Z_{\tau} J Z_{\tau}^{-1}=J$. This gives immediatly that $X_{0}\left(I_{0}^{\prime}\right)=\{0\}$, and so $X_{0}$ is well defined in $\mathcal{A}_{0}^{\prime \mathcal{J}}$. Now the proof that $X_{0}$ is an isomorphism is very similar to that of Proposition 2.21 , with the inverse being defined as $X_{0}^{\prime}(A)=\Phi_{0}^{\mathcal{J}}(A)$

2.5.3 The local algebras $\mathcal{A}_{t}^{\prime \mathcal{J}}\left(t \in \mathbb{R}^{+}\right)$. To identify these local algebras we will eliminate the flip by doubling the dimension (see [18: Scheme 3.3]).

Lemma 2.27. Let $\mathcal{X}$ be a $C^{*}$-algebra with identity e whose center contains a selfadjoint projection $p$. Let $\mathcal{Y}$ be generated by $\mathcal{X}$ and a selfadjoint flip $j$ with the properties $j \mathcal{X} j \subset \mathcal{X}$ and, in particular, $j p j=e-p$. Then any element $a$ of $\mathcal{Y}$ can be written uniquely as sum $a_{1}+a_{2} j$ with $a_{1}, a_{2} \in \mathcal{X}$, and the mapping $L: \mathcal{Y} \rightarrow[p \mathcal{X} p]^{2 \times 2}$ defined by

$$
a \longmapsto\left[\begin{array}{ll}
p a_{1} p & p a_{2} p \\
p \tilde{a}_{2} p & p \tilde{a}_{1} p
\end{array}\right]
$$

with $\tilde{a}=j a j$ is an isometric *-isomorphism. 
Proof. In [18: Scheme 3.3] it was already shown that $L$ is an isometric continuous homomorphic embedding. We have only to prove that it is onto. Let $a_{i}(i=1, \ldots, 4)$ be any four elements of $\mathcal{X}$. If we define

$$
a=p a_{1} p+p a_{2} j(e-p)+(e-p) j a_{3} p+(e-p) \tilde{a}_{4}(e-p)
$$

then $a \in \mathcal{Y}$, and it easy to see that $L(a)=\left[\begin{array}{ll}p a_{1} p & p a_{2} p \\ p a_{3} p & p a_{4} p\end{array}\right]$ which proves that $L$ is onto

So let $f_{t}$ be a continuous function with support in $\mathbb{R}^{+}$such that $f_{t}(t)=1$, and put $p=\Phi_{t}^{\mathcal{J}}\left(f_{t}\right), j=\Phi_{t}^{\mathcal{J}}(J)$ and $e=\Phi_{t}^{\mathcal{J}}(I)$. We have that $p^{2}=p, p$ commutes with all the algebra generators except $j$, and $j p j=e-p$. Any element $a \in \mathcal{A}_{t}^{\prime J}$ can be written (due to the properties of $J$ ) as $a=a_{1}+a_{2} j$, where $a_{1}$ and $a_{2}$ belongs to the algebra without the flip. It is then possible to apply Lemma 2.27 .

Define now the homomorphism

$$
\dot{X}_{t}: \mathcal{A}_{t}^{\prime \mathcal{J}} \rightarrow\left[\operatorname{alg}\left(I, \chi_{+}, \stackrel{\mathscr{W}}{\left(\chi_{+}\right)}\right)\right]^{2 \times 2}, \quad \dot{X}_{t}=X_{t} L
$$

where $X_{t}$ represents now the canonical (diagonal) extension for matrix operators of the strong limit defined in (42). We have then the following

Proposition 2.28. The local algebra $\mathcal{A}_{t}^{\prime J}\left(t \in \mathbb{R}^{+}\right)$is isomorphic to

$$
\left[\operatorname{alg}\left(I, \chi_{+}, \stackrel{\circ}{W}\left(\chi_{+}\right)\right]^{2 \times 2}\right.
$$

and the isomorphism is given by $\dot{X}_{t}$. In particular,

$$
\begin{aligned}
& \dot{X}_{t}\left(\Phi_{\imath}^{\mathcal{J}}\left(P_{r}\right)\right)=\left[\begin{array}{ll}
I & 0 \\
0 & I
\end{array}\right] \\
& \dot{X}_{t}\left(\Phi_{t}^{\mathcal{J}}(\stackrel{W}{W}(a))\right)= \\
& {\left[\begin{array}{cc}
a(-\infty) \dot{W}\left(\chi_{-}\right)+a(+\infty) \dot{W}\left(\chi_{+}\right) & 0 \\
0 & a(+\infty) \dot{W}\left(\chi_{-}\right)+a(-\infty) \dot{W}\left(\chi_{+}\right)
\end{array}\right]} \\
& \dot{X}_{t}\left(\Phi_{t}^{J}(c I)\right)=\left[\begin{array}{cc}
c\left(t^{-}\right) \chi_{-}+c\left(t^{+}\right) \chi_{+} & 0 \\
0 & c\left(-t^{+}\right) \chi_{-}+c\left(-t^{-}\right) \chi_{+}
\end{array}\right] \\
& \dot{X}_{t}\left(\Phi_{t}^{\mathcal{J}}(J)\right)=\left[\begin{array}{ll}
0 & I \\
I & 0
\end{array}\right] .
\end{aligned}
$$

Proof. To prove the specific values of $\dot{X}_{t}$, use Lemma 2.27 together with the proposition corresponding to Proposition 2.14 (for $\Phi_{t}^{\mathcal{J}}\left(P_{\tau}\right)$ ), and note that $Z_{\tau} \tilde{V}_{-t} \dot{W}^{\circ}(a) \tilde{V}_{t} Z_{r}^{-1}$ $=\stackrel{W}{W}\left(a_{r}\right)$ with $a_{r}(\xi)=a(\xi \tau)$, and that $\left(Z_{\tau} \tilde{V}_{-t} c \tilde{V}_{t} Z_{r}^{-1}\right)(x)=c\left(\frac{x}{\tau}+t\right)$. These values imply that $\dot{X}_{t}\left(I_{t}^{\prime}\right)=\{0\}$, and so $\dot{X}_{t}$ is well defined in $\mathcal{A}_{t}^{\prime J}$. We have only to prove now that invertibility in $\operatorname{Im}\left(\dot{X}_{t}\right)$ is equivalent to invertibility in $\mathcal{A}_{t}^{\prime J}$. As $\dot{X}_{t}$ is a unital homomorphism, if $\Phi_{t}^{\mathcal{J}}\left(A_{\tau}\right)$ is invertible in $\mathcal{A}_{t}^{\prime \mathcal{J}}$, it easy to see that $\dot{X}_{t}\left(\Phi_{t}^{\mathcal{J}}\left(A_{\tau}\right)\right)$ is invertible, 
and due to the inverse closedness, the inverse must belong to $\left[\operatorname{alg}\left(I, \chi_{+}, \stackrel{W}{W}\left(\chi_{+}\right)\right]^{2 \times 2}\right.$. To prove the converse define the application

$$
\begin{aligned}
& \dot{X}_{t}^{\prime}:\left[\operatorname{alg}\left(I, \chi_{+}, \stackrel{W}{W}\left(\chi_{+}\right)\right]^{2 \times 2} \rightarrow \mathcal{A}_{t}^{\prime \mathcal{J}}\right. \\
& \dot{X}_{t}^{\prime}\left(\left[\begin{array}{ll}
A_{11} & A_{12} \\
A_{21} & A_{22}
\end{array}\right]\right)=L^{-1}\left(\left[\begin{array}{ll}
p \Phi_{t}^{\mathcal{J}}\left(\tilde{V}_{t} A_{11} \tilde{V}_{-t}\right) & p \Phi_{t}^{\mathcal{J}}\left(\tilde{V}_{t} A_{12} \tilde{V}_{-t}\right) \\
p \Phi_{t}^{\mathcal{J}}\left(\tilde{V}_{t} A_{21} \tilde{V}_{-t}\right) & p \Phi_{t}^{\mathcal{J}}\left(\tilde{V}_{t} A_{22} \tilde{V}_{-t}\right)
\end{array}\right]\right) .
\end{aligned}
$$

This application is a unital homomorphism, and in a way similar to the proof of Proposition 2.21 it can be shown that

$$
\dot{X}_{t}^{\prime}\left(\dot{X}_{t}\left(\Phi_{t}^{\mathcal{J}}\left(A_{\tau}\right)\right)\right)=\Phi_{t}^{J}\left(A_{\tau}\right)
$$

and so invertibility in $\operatorname{Im}\left(\dot{X}_{t}\right)$ implies invertibility in $\mathcal{A}_{t}^{\prime \mathcal{J}}$

2.5.4 Second localization. As before, the local algebra at the point infinity is still too complex to identify but possesses a non trivial center.

Proposition 2.29. The cosets $\Phi_{\infty}^{\mathcal{J}}(\dot{W}(f))$ with $f \in \tilde{C}(\dot{\mathbb{R}})$ are in the center of $\mathcal{A}_{\infty}^{\prime \mathcal{J}}$.

Proof. It is almost immediate that ${ }^{\circ}(f) J=J W(f)$. The other results have the same proof as those in Proposition 2.16

This last result means that we can again localize $\mathcal{A}_{\infty}^{\prime J}$ over the maximal ideal space of the subalgebra $\mathcal{C}$ ' generated by these cosets. This maximal ideal space is now formed by the cosets $\Phi_{\infty}^{\mathcal{J}}\left(\mathscr{D}^{\circ}\left(f_{x}\right)\right)$ with $f_{x}(x)=f_{x}(-x)=0$ and $x \in[0, \infty]$, and it is isomorphic to $[0, \infty]$.

In order to apply the local principle of Allan, let $I_{\infty, x}^{\prime}(x \in[0, \infty])$ be the smallest closed two-sided ideal of $\mathcal{A}_{\infty}^{\prime \mathcal{J}}$ which contains the ideal $x$ of $\mathcal{C}$ '. We call $\Phi_{\infty, x}^{\mathcal{J}}$ the homomorphism which is the composition of the canonical homomorphism from $\mathcal{A}_{\infty}^{\prime \mathcal{J}}$ to $\mathcal{A}_{\infty, x}^{\prime \mathcal{J}}:=\mathcal{A}_{\infty}^{\prime \mathcal{J}} / I_{\infty, x}^{\prime}$, with $\Phi_{\infty}^{\mathcal{J}}$. We have again a lemma that identifies some elements of $I_{\infty, x}^{\prime}$ and whose proof, due to being almost the same as that of Lemma 2.17, we omit. $=0$.

Lemma 2.30. If $a \in P C(\dot{\mathbb{R}})$ is continuous at $\pm x$ and $a( \pm x)=0$, then $\Phi_{\infty, x}^{\mathcal{J}}(\stackrel{\circ}{W}(a))$

2.5.5 Identification of the local algebras. Here, we will use again the strong limits $W_{s}$ defined in (29), but the only algebra in which we can apply the homomorphism directly is $\mathcal{A}_{\infty, 0}^{\prime \mathcal{J}}$, because only $W_{0}$ is well defined (i.e. the strong limit exists) when applied to the flip $J$.

2.5.6 The local algebra $\mathcal{A}_{\infty, 0}^{\prime \mathcal{J}}$. If we define the algebra $\mathcal{B}^{\prime}$ as $\operatorname{alg}\left(I, P_{1}, \chi_{+}, \stackrel{\mathscr{W}}{ }\left(\chi_{+}\right), J\right)$, the strong limit $W_{0}$ is an algebra homomorphism between the algebras $\mathcal{A}^{\prime}$ and $\mathcal{B}^{\prime}$ and the following theorem, with a proof equal to the case without the flip (Proposition 2.21) assures that $W_{0}$ is also an isomorphism. 
Proposition 2.31. The local algebra $\mathcal{A}_{\infty, 0}^{\prime J}$ is isomorphic to the algebra $\mathcal{B}^{\prime}$, and the isomorphism is given by $W_{0}$.

2.5.7 The local algebra $\mathcal{A}_{\infty, s}^{\prime \mathcal{J}}\left(s \in \mathbb{R}^{+}\right)$. We will here again apply Lemma 2.27 to eliminate the flip by doubling the dimension. So let $f_{s}$ be a continuous function with support in $\mathbb{R}^{+}$such that $f_{s}(s)=1$, and put $p=\Phi_{\infty, s}^{\mathcal{J}}\left(\dot{W}\left(f_{s}\right)\right), j=\Phi_{\infty, s}^{\mathcal{J}}(J)$ and $e=\Phi_{\infty, s}^{\mathcal{J}}(I)$. We have that $p^{2}=p, p$ commutes with all the algebra generators except $j$, and $j p j=e-p$. Any element $a \in \mathcal{A}_{\infty, \mathrm{J}}^{\prime \mathcal{J}}$ can be written (due to the properties of $J)$ as $a=a_{1}+a_{2} j$, where $a_{1}$ and $a_{2}$ belong to the algebra without the flip. It is then possible to apply Lemma 2.27. Define the homomorphism

$$
\dot{W}_{s}: \mathcal{A}_{\infty, s}^{\prime J} \rightarrow\left[\operatorname{alg}\left(I, \chi_{+}, \dot{W}\left(\chi_{+}\right), P_{1}\right)\right]^{2 \times 2}, \quad \dot{W}_{s}=W_{s} L
$$

where $W$, represents the canonical extension for matrix operators of the strong limit defined in (29). We have then the following

Proposition 2.32. The algebra $\mathcal{A}_{\infty, s}^{\prime J}\left(s \in \mathbb{R}^{+}\right)$is isomorphic to

$$
\left[\operatorname{alg}\left(I, P_{1}, \chi_{+}, \dot{W}\left(\chi_{+}\right)\right]^{2 \times 2},\right.
$$

and the isomorphism is given by $\dot{W}_{s}$. In particular,

$$
\begin{aligned}
& \dot{W}_{s}\left(\Phi_{\infty, s}^{\mathcal{J}}\left(P_{r}\right)\right)=\left[\begin{array}{cc}
P_{1} & 0 \\
0 & P_{1}
\end{array}\right] \\
& \dot{W}_{s}\left(\Phi_{\infty, s}^{\mathcal{J}}(\dot{W}(a))\right)= \\
& {\left[\begin{array}{ccc}
a\left(s^{-}\right) \dot{W}\left(\chi_{-}\right)+a\left(s^{+}\right) \dot{W}\left(\chi_{+}\right) & 0 \\
0 & a\left(-s^{+}\right) \dot{W}^{\circ}\left(\chi_{-}\right)+a\left(-s^{-}\right) \dot{W}\left(\chi_{+}\right)
\end{array}\right] } \\
& \dot{W}_{s}\left(\Phi_{\infty, s}^{\mathcal{J}}(c I)\right)=\left[\begin{array}{cc}
c(-\infty) \chi_{-}+c(+\infty) \chi_{+} & 0 \\
0 & c(+\infty) \chi_{-}+c(-\infty) \chi_{+}
\end{array}\right] \\
& \dot{W}_{s}\left(\Phi_{\infty, s}^{\mathcal{J}}(J)\right)=\left[\begin{array}{ll}
0 & I \\
I & 0
\end{array}\right] .
\end{aligned}
$$

Proof. First we must see that $\dot{W}_{s}$ is well defined in $\mathcal{A}_{\infty, s}^{\prime J}$. Having already the results from Lemma 2.18 one can easily see that $\dot{W}_{s}\left(I_{\infty}^{\prime}\right)$ and $\dot{W}_{s}\left(I_{\infty, s}^{\prime}\right)$ are $\{0\}$. The computation of the formulas (50) - (53) is easy by the use of the results in Lemma 2.18. We shall only prove now that invertibility in $\operatorname{Im}\left(\dot{W}_{s}\right)$ is equivalent to invertibility in $\mathcal{A}_{\infty, s}^{\prime \mathcal{J}}$. As $\dot{W}_{s}$ is a unital homomorphism, if $\Phi_{\infty, s}^{\mathcal{J}}\left(A_{r}\right)$ is invertible in $\mathcal{A}_{\infty, s}^{\prime J}$, then $\dot{W}_{s}\left(\Phi_{\infty, s}^{\mathcal{J}}\left(A_{\tau}\right)\right)$ is invertible. To prove the converse define the application

$$
\begin{aligned}
& \dot{W}_{s}^{\prime}: {\left[\operatorname{alg}\left(I, P_{1}, \chi_{+}, \dot{W}\left(\chi_{+}\right)\right]^{2 \times 2} \rightarrow \mathcal{A}_{\infty, s}^{\mathcal{J}}\right.} \\
& \dot{W}_{s}^{\prime}\left(\left[\begin{array}{ll}
A_{11} & A_{12} \\
A_{21} & A_{22}
\end{array}\right]\right) \\
& \quad=L^{-1}\left(\left[\begin{array}{ll}
p \Phi_{\infty, s}^{\mathcal{J}}\left(U_{s} Z_{\tau} A_{11} Z_{\tau}^{-1} U_{-s}\right) & p \Phi_{\infty, s}^{\mathcal{J}}\left(U_{s} Z_{\tau} A_{12} Z_{\tau}^{-1} U_{-s}\right) \\
p \Phi_{\infty, s}^{\mathcal{J}}\left(U_{s} Z_{r} A_{21} Z_{\tau}^{-1} U_{-s}\right) & p \Phi_{\infty, s}^{\mathcal{J}}\left(U, Z_{\tau} A_{22} Z_{\tau}^{-1} U_{-s}\right)
\end{array}\right]\right) .
\end{aligned}
$$


This application is a unital homorphism, and in a similar way to the proof of Proposition 2.21 it can be shown that

$$
\dot{W}_{s}^{\prime}\left(\dot{W}_{s}\left(\Phi_{\infty, s}^{\mathcal{J}}\left(A_{\tau}\right)\right)\right)=\Phi_{\infty, s}^{\mathcal{J}}\left(A_{\tau}\right)
$$

and so invertibility in $\operatorname{Im}\left(\dot{W}_{0}\right)$ implies invertibility in $\mathcal{A}_{\infty, s}^{\prime \mathcal{J}}$

2.5.8 The local algebra $\mathcal{A}_{\infty, \infty}^{\prime \mathcal{J}}$. For this local algebra we have no locally equivalent representation given by strong limits, and so the algebra must be studied by properties of its generators.

Proposition 2.33. The local algebra $\mathcal{A}_{\infty, \infty}^{\prime J}$ is generated by the elements

$$
\begin{aligned}
e & =\Phi_{\infty, \infty}^{\mathcal{J}}(I) \\
p_{1} & =\Phi_{\infty, \infty}^{\mathcal{J}}\left(P_{r}\right), \quad p_{2}=\Phi_{\infty, \infty}^{\mathcal{J}}\left(\stackrel{\mathscr{W}}{\mathcal{J}}\left(\chi_{+}\right)\right), \quad p_{3}=\Phi_{\infty, \infty}^{\mathcal{J}}\left(\chi_{+}\right) \\
j & =\Phi_{\infty, \infty}^{\mathcal{J}}(J) .
\end{aligned}
$$

Proof. We have that

$$
\begin{aligned}
\Phi_{\infty, \infty}^{\mathcal{J}}(c I)= & \Phi_{\infty, \infty}^{\mathcal{J}}\left(c(-\infty) \chi_{-}+c(+\infty) \chi_{+}\right) \\
& -\Phi_{\infty, \infty}^{\mathcal{J}}\left(-\left(c-c(-\infty) \chi_{-}-c(+\infty) \chi_{+}\right) I\right),
\end{aligned}
$$

and as $c-c(-\infty) \chi_{-}-c(+\infty) \chi_{+}$is a function continuous at infinity and taking the value 0 there, by Lemma 2.13 ,

$$
\Phi_{\infty, \infty}^{\mathcal{J}}\left(\left(c-c(-\infty) \chi_{-}-c(+\infty) \chi_{+}\right) I\right)=0 .
$$

Using the same reasoning,

$$
\Phi_{\infty, \infty}^{\mathcal{J}}(\stackrel{\mathscr{W}}{(a)})=\Phi_{\infty, \infty}^{\mathcal{J}}\left(a(-\infty) \stackrel{\circ}{W}\left(\chi_{-}\right)+c(+\infty) \stackrel{\circ}{W}\left(\chi_{+}\right)\right)
$$

For the other generators, the result is obvious

We have then an algebra generated by the identity and four idempotents. The nontrivial relations between these generators are given in the next proposition. The only relation that does not come directly from the already known relations in the algebra $\mathcal{A}$ is $p_{2} p_{3}=p_{3} p_{2}$, or $\Phi_{\infty, \infty}^{\mathcal{J}}\left(\stackrel{W}{W}\left(\chi_{+}\right) \chi_{+}\right)=\Phi_{\infty, \infty}^{\mathcal{J}}\left(\chi_{+} W^{\circ}\left(\chi_{+}\right)\right)$. The proof in this case is the same as in Proposition 2.22.

Proposition 2.34. The following relations hold:

- $j p_{1} j=p_{1}$ and $p_{1} p_{3}=p_{3} p_{1}$.

- $j p_{2} j=e-p_{2}$ and $p_{2} p_{3}=p_{3} p_{2}$.

- $j p_{3} j=e-p_{3}$.

As the projection $p_{3}$ commutes with $p_{1}$ and $p_{2}$, and $j p_{3} j=e-p_{3}$, we are in the position again to apply Lemma 2.27 and to eliminate the flip by doubling the dimension. 
This means, we have an isomorphism $L: \mathcal{A}_{\infty, \infty}^{\prime \mathcal{J}} \rightarrow \mathcal{D}^{2 \times 2}$, with $\mathcal{D}=\operatorname{alg}\left(p_{3}, p_{1} p_{3}, p_{2} p_{3}\right)$, whose image for the generators of $\mathcal{A}_{\infty, \infty}^{\mathcal{J}}$ are

$$
\begin{aligned}
L(e) & =\left[\begin{array}{cc}
p_{3} & 0 \\
0 & p_{3}
\end{array}\right] \\
L\left(p_{1}\right) & =\left[\begin{array}{cc}
p_{1} p_{3} & 0 \\
0 & p_{1} p_{3}
\end{array}\right], \quad L\left(p_{2}\right)=\left[\begin{array}{cc}
p_{2} p_{3} & 0 \\
0 & \left(e-p_{2}\right) p_{3}
\end{array}\right], \quad L\left(p_{3}\right)=\left[\begin{array}{cc}
p_{3} & 0 \\
0 & 0
\end{array}\right] \\
L(j) & =\left[\begin{array}{cc}
0 & p_{3} \\
p_{3} & 0
\end{array}\right],
\end{aligned}
$$

and we can see, because $p_{3}$ is the identity for $\mathcal{D}$, that we are in the situation of an algebra generated by the identity and two idempotents. As before, the two projections theorem gives us a symbol mapping $N$, to a space of matrix functions:

$$
\begin{aligned}
N\left(p_{3}\right): x & \longmapsto\left[\begin{array}{ll}
1 & 0 \\
0 & 1
\end{array}\right] \\
N\left(p_{1} p_{3}\right): x & \longmapsto\left[\begin{array}{ll}
1 & 0 \\
0 & 0
\end{array}\right] \\
N\left(p_{2} p_{3}\right): x & \longmapsto\left[\begin{array}{cc}
x & \sqrt{x(1-x)} \\
\sqrt{x(1-x)} & 1-x
\end{array}\right]
\end{aligned}
$$

with $x \in \sigma_{\mathcal{D}}\left(p_{1} p_{3} p_{2} p_{3} p_{1}\right)$, if only

$$
\{0,1\} \subset \sigma_{\mathcal{D}}\left(p_{1} p_{3} p_{2} p_{3} p_{1}\right)=\sigma_{\mathcal{D}}\left(\Phi_{\infty, \infty}^{\mathcal{J}}\left(P_{\tau} \chi_{+} \stackrel{ }{W}\left(\chi_{+}\right) \chi_{+} P_{+}\right)\right)
$$

So we only need to find this local spectrum.

Proposition 2.35. We have $\sigma_{\mathcal{D}}\left(\Phi_{\infty, \infty}^{\mathcal{J}}\left(P_{\tau} \chi_{+} \stackrel{\mathscr{W}}{ }\left(\chi_{+}\right) \chi_{+} P_{r}\right)\right)=[0,1]$.

Proof. By the same arguments as in the case of the algebra without the flip, one can see that

$$
\sigma_{\mathcal{D}}\left(\Phi_{\infty, \infty}^{\mathcal{J}}\left(P_{\tau} \chi_{+} \stackrel{\circ}{W}\left(\chi_{+}\right) \chi_{+} P_{r}\right)\right) \subset[0,1]
$$

To prove the other inclusion suppose that $\lambda \in(0,1)$ and

$$
\Phi_{\infty, \infty}^{\mathcal{J}}\left(\lambda \chi_{+}-P_{\tau} \chi_{+} \stackrel{\circ}{W}\left(\chi_{+}\right) \chi_{+} P_{r}\right)
$$

is invertible in $\mathcal{D}$. Then it is not difficult to see that $\Phi_{\infty, \infty}^{\mathcal{J}}\left(\lambda I-P_{r} \chi_{+} \stackrel{\circ}{W}\left(\chi_{+}\right) \chi_{+} P_{\tau}\right)$ is invertible in $\mathcal{A}_{\infty, \infty}^{\prime \mathcal{J}}$. Now define the function $\chi_{+}^{\prime}$, continuous on $\mathbb{R}$, with $\chi_{+}^{\prime}(-\infty)=0$ and $\chi_{+}^{\prime}(+\infty)=1$, and such that the imaginary part of $\chi_{+}^{\prime}(x)$ is greater than zero for $x \in \mathbb{R}$. We have that, for $A_{\tau}=\lambda I-P_{\tau} \chi_{+}^{\prime} \dot{W}\left(\chi_{+}^{\prime}\right) \chi_{+}^{\prime} P_{r}$,

$$
\Phi_{\infty, \infty}^{\mathcal{J}}\left(\lambda I-P_{\tau} \chi_{+} \stackrel{\circ}{W}\left(\chi_{+}\right) \chi_{+} P_{r}\right)=\Phi_{\infty, \infty}^{\mathcal{J}}\left(A_{r}\right),
$$

and $\dot{W}_{s}\left(A_{r}\right)\left(s \in \mathbb{R}^{+}\right)$as well as $W_{0}\left(A_{r}\right)$ are invertible. As also $W^{0}\left(\left(A_{\tau}\right)\right)$ is a Fredholm operator (see [6] or [17: Section 15]) we have that $A_{r}+\mathcal{J}$ is invertible in $\mathcal{A}^{\prime \mathcal{J}}$. But this is a contradiction since, by Proposition 2.23, $A_{\tau}+\mathcal{J}$ is not invertible in $\mathcal{A}^{\mathcal{J}}$ and $\mathcal{A}^{\mathcal{J}}$ is $a^{*}$-subalgebra of $\mathcal{A}^{\prime} \mathcal{J}$. So we proved that the open interval $(0,1)$ must be contained in the spectrum, and as the spectrum must be closed the result follows 
To synthesize our results, let $M^{\prime}=N L$ and $M=M^{\prime} \Phi_{\infty, \infty}^{\mathcal{J}}$. Let $\mathcal{Z}$ be the closed subalgebra of the $(4 \times 4)$ - matrix functions defined in the interval $[0,1]$ generated by the elements

$$
\begin{aligned}
& e^{\prime}: x \longmapsto\left[\begin{array}{llll}
1 & 0 & 0 & 0 \\
0 & 1 & 0 & 0 \\
0 & 0 & 1 & 0 \\
0 & 0 & 0 & 1
\end{array}\right] \\
& p_{1}^{\prime}: x \longmapsto\left[\begin{array}{llll}
1 & 0 & 0 & 0 \\
0 & 0 & 0 & 0 \\
0 & 0 & 1 & 0 \\
0 & 0 & 0 & 0
\end{array}\right] \\
& p_{2}^{\prime}: x \longmapsto\left[\begin{array}{cccc}
x & \sqrt{x(1-x)} & 0 & 0 \\
\sqrt{x(1-x)} & 1-x & 0 & 0 \\
0 & 0 & 1-x & -\sqrt{x(1-x)} \\
0 & 0 & -\sqrt{x(1-x)} & x
\end{array}\right] \\
& p_{3}^{\prime}: x \longmapsto\left[\begin{array}{llll}
1 & 0 & 0 & 0 \\
0 & 1 & 0 & 0 \\
0 & 0 & 0 & 0 \\
0 & 0 & 0 & 0
\end{array}\right] \\
& j^{\prime}: x \longmapsto\left[\begin{array}{llll}
0 & 0 & 1 & 0 \\
0 & 0 & 0 & 1 \\
1 & 0 & 0 & 0 \\
0 & 1 & 0 & 0
\end{array}\right] \text {. }
\end{aligned}
$$

We proved the following proposition.

Proposition 2.36. The local algebra $\mathcal{A}_{\infty, \infty}^{\prime J}$ is isometrically isomorphic to the algebra $\mathcal{Z}$, and the isomorphism is given by $M^{\prime}$. The coset $\Phi_{\infty, \infty}^{\mathcal{J}}\left(A_{r}\right)$ is invertible in the local algebra $\mathcal{A}_{\infty, \infty}^{\prime J}$ if and only if $M\left(A_{\tau}\right)$ is invertible in $\mathcal{Z}$. In particular, we have:

- $M(c I)=c(-\infty)\left(e^{\prime}-p_{3}^{\prime}\right)+c(+\infty) p_{3}^{\prime}$.

- $M(\stackrel{W}{W}(a))=a(-\infty)\left(e^{\prime}-p_{2}^{\prime}\right)+a(+\infty) p_{2}^{\prime}$.

- $M(J)=j^{\prime}$.

- $M\left(P_{r}\right)=p_{1}^{\prime}$.

2.6 The main theorem. Having identified all the local algebras, we can now state the main result. The detailed proof of this result is written above (it is the subject of this whole section), and so below we just give a sketch of the proof. 
Theorem 2.37. Let $A$ be any operator from the subalgebra of $\mathcal{L}\left(L^{2}(\mathbb{R})\right)$ generated by the operators $c I$ and $\dot{W}(a)$, with $a, c \in P C(\dot{\mathbb{R}})$, and by the fip operator $J$, defined by $(J u)(x)=u(-x)$. Then the approximation method

$$
\left(P_{\tau} A P_{\tau}+Q_{\tau}\right) u_{\tau}=f
$$

can be used to get an approximate solution to the equation

$$
A u=f
$$

(i.e. the method applies to $A$ ) if and only if $A, \tilde{A}, W_{0}\left(A_{\tau}\right), M\left(A_{\tau}\right)$ and $\dot{W}_{s}\left(A_{\tau}\right)$ (s $\in$ $\mathbb{R}^{+}$) are invertible, with $A_{\tau}=P_{\tau} A P_{\tau}+Q_{\tau}$.

Proof. By Theorems 2.1 and 2.4, the applicability of the approximation method is equivalent to the invertibility of the operators $A$ and $\tilde{A}$, and the invertibility of the $\operatorname{coset}\left(A_{\tau}\right)+\mathcal{J}$ in $\mathcal{A}^{\prime} \mathcal{J}$. The cosets $(f I)+\mathcal{J}$ with $f \in \tilde{C}(\dot{\mathbb{R}})$ are in the center of $\mathcal{A}^{\prime} \mathcal{J}$, and applying the local principle of Allan, instead of an invertibility problem in $\mathcal{A}^{\prime \mathcal{J}}$, we have now invertibility problems in each of the (simpler) local algebras $\mathcal{A}_{t}^{\prime J} \quad(t \in[0,+\infty])$. All the local algebras except the local algebra at infinity are identificable, and if $A$ is a Fredholm operator, then the cosets $\Phi_{t}^{\mathcal{J}}\left(A_{\tau}\right) \quad\left(t \in \mathbb{R}_{0}^{+}\right)$are invertible (see Propositions 2.26 and 2.28). Regarding the local algebra $\mathcal{A}_{\infty}^{\prime \mathcal{J}}$, it has the cosets $\Phi_{\infty}^{\mathcal{J}}\left(W^{\circ}(f)\right) \quad(f \in$ $\tilde{C}(\dot{\mathbb{R}}))$ in its center. Applying again the local principle of Allan, we are able to identify all the local algebras through the isomorphisms $W_{0}$ (for the local algebra at the point 0 see Proposition 2.31), $M$ (for the local algebra at the point infinity see Proposition 2.36), and $\dot{W}_{s}$ (for the local algebras corresponding to the points $s \in \mathbb{R}^{+}$see Proposition 2.32 ), obtaining in this way the aimed result

2.7 The system case. Formaly, we proved Theorem 2.37 for the scalar case only. But if we consider $a$ or $c$ in $[P C(\dot{\mathbb{R}})]^{n \times n}$, the proofs remain the same. This covers the operators related with systems of singular integral equations or systems of WienerHopf operators. Obviously, the operators that result from the homomorphisms have then matrix coefficients, and it can be difficult in the general case to find invertibility conditions for these operators. For a non-scalar version of the two projections theorem see [7].

\section{Examples}

We will continue our exposition on the finite section method by presenting two examples of application of Theorem 2.37. The first is about singular integral operators, where the results concerning the finite section method are already known (see, for example, [9]), and the other one is the application to an operator of Wiener-Hopf plus Hankel type, which appears here for the first time. In what follows the symbols $P_{1}$ and $Q_{1}$ can also represent the matrix operators with $P_{1}$ and $Q_{1}$ in the main diagonal and zero elsewhere, when needed.

3.1 Singular integral operators. Consider the operator

$$
A=c_{1} \stackrel{\circ}{W}\left(\chi_{-}\right)+c_{2} \stackrel{\circ}{W}\left(\chi_{+}\right)
$$


with $c_{1}, c_{2} \in P C(\dot{\mathbb{R}})$. It is usually called a paired singular integral operator, as it can be written in the form

$$
\frac{c_{1}+c_{2}}{2} I+\frac{c_{1}-c_{2}}{2} S_{\mathbb{R}}, \quad\left(S_{\mathbb{R}} u\right)(t)=\frac{1}{\pi i} \int_{\mathbb{R}} \frac{u(s)}{s-t} d s .
$$

Applying Theorem 2.37 to $A$ we obtain the following result.

Corollary 3.1. The approximation method (1) applies to the operator $A$ in (66) if and only if the operator $A$ is invertible and the point 0 is not contained in the area limited by the triangle defined by the points $1, \frac{c_{1}(-\infty)}{c_{2}(-\infty)}$ and $\frac{c_{1}(+\infty)}{c_{2}(+\infty)}$.

Proof. The direct use of Theorem 2.37 leads to the following operators (resp. operator functions) that must be invertible, in order to guarantee the applicability of the approximation method:

a) $A=c_{1} \dot{W}\left(\chi_{-}\right)+c_{2} \dot{W}\left(\chi_{+}\right)$.

b) $\tilde{A}=\left(c_{1}(-\infty) \chi_{-}+c_{1}(+\infty) \chi_{+}\right) \stackrel{\circ}{W}\left(\chi_{+}\right)+\left(c_{2}(-\infty) \chi_{-}+c_{2}(+\infty) \chi_{+}\right) \stackrel{W}{W}\left(\chi_{-}\right)$.

c) $W_{0}\left(A_{r}\right)=P_{1}\left(\left(c_{1}(-\infty) \chi_{-} c+c_{1}(+\infty) \chi_{+}\right) \grave{W}\left(\chi_{-}\right)\right.$ $\left.+\left(c_{2}(-\infty) \chi_{-}+c_{2}(+\infty) \chi_{+}\right) \stackrel{\circ}{W}\left(\chi_{+}\right)\right) P_{1}+Q_{1}$.

d) $\dot{W}_{s}\left(A_{\tau}\right)=P_{1}\left[\begin{array}{cc}\left(c_{2}(-\infty) \chi_{-}+c_{2}(+\infty) \chi_{+}\right) & 0 \\ 0 & \left(c_{1}(+\infty) \chi_{-}+c_{1}(-\infty) \chi_{+}\right)\end{array}\right] P_{1}+Q_{1}$.

e) $M\left(A_{\tau}\right): x \longmapsto\left[\begin{array}{cccc}c_{1}(+\infty)(1-x)+c_{2}(+\infty) x & 0 & 0 & 0 \\ 0 & 1 & 0 & 0 \\ 0 & 0 & c_{1}(-\infty) x+c_{2}(-\infty)(1-x) & 0 \\ 0 & 0 & 0 & 1\end{array}\right]$ for $x \in[0,1]$.

The fact that $A$ must be invertible implies immediatly the invertibility of $\tilde{A}$ and that $c_{1,2}( \pm \infty)$ are different from zero, which also means that $\dot{W}_{s}\left(A_{\tau}\right)$ are invertible. It is also not difficult to see that the invertibility of $W_{0}\left(A_{\tau}\right)$ implies the invertibility of $M\left(A_{\tau}\right)$ Finally writing $W_{0}\left(A_{\tau}\right)$ as the operator

$$
a^{\prime} \stackrel{\circ}{W}\left(\chi_{-}\right)+\stackrel{\circ}{W}\left(\chi_{+}\right), \quad \text { with } a^{\prime}(x)= \begin{cases}\frac{c_{1}(+\infty)}{c_{2}(+\infty)} & \text { if } 0<x<1 \\ \frac{c_{1}(-\infty)}{c_{2}(-\infty)} & \text { if }-1<x<0 \\ 1 & \text { if }|x|>1\end{cases}
$$

and applying usual invertibility conditions for singular integral operators (see [11]) it is obtained that the point 0 must not be contained in the area limited by the triangle defined by the points $1, \frac{c_{1}(-\infty)}{c_{2}(-\infty)}$ and $\frac{c_{1}(+\infty)}{c_{2}(+\infty)}$

We can consider also a more complex type of paired operator. Let $A$ be the operator

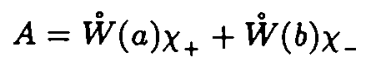

with $a, b \in P C(\dot{\mathbb{R}})$. The following result is then obtained. 
Corollary 3.2. The approximation method (1) applies to the operator $A$ in (67) if and only if the following conditions are satisfied:

a) The operator $A$ is invertible.

b) The operator $\chi_{+} \dot{W}(\tilde{a}) \chi_{+}+\chi_{-} \dot{W}(\tilde{b}) \chi_{-}$is invertible.

c) For any $s \in \mathbb{R}$, the point 0 is not contained in the area limited by the triangle defined by the points $1, \frac{a\left(s^{-}\right)}{a\left(s^{+}\right)}$and $\frac{b\left(s^{-}\right)}{b\left(s^{+}\right)}$.

Proof. As the operator $A$ belongs to the algebra without the flip we can apply the more simple homomorphisms $W_{s}$ instead of $\stackrel{k}{W}$. A necessary and sufficient condition for the applicability of the finite section method is the invertibility of the following operators (resp. operator functions):

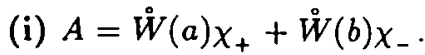

(ii) $\tilde{A}=\chi_{+} \dot{W}(\tilde{a}) \chi_{+}+\chi_{-} \stackrel{W}{W}(\tilde{b}) \chi_{-}$.

(iii) $W_{s}\left(A_{\tau}\right)=P_{1}\left(\stackrel{\circ}{W}\left(\chi_{-}\right)\left(a\left(s^{-}\right) \chi_{+}+b\left(s^{-}\right) \chi_{-}\right)+\stackrel{\circ}{W}\left(\chi_{+}\right)\left(a\left(s^{+}\right) \chi_{+}+b\left(s^{+}\right) \chi_{-}\right)\right) P_{1}+Q_{1}$ for $s \in \mathbb{R}$.

(iv) $M\left(A_{\tau}\right): x \longmapsto\left[\begin{array}{cccc}a(-\infty)(1-x)+a(+\infty) x & 0 & 0 & 0 \\ 0 & 1 & 0 & 0 \\ 0 & 0 & b(-\infty) x+b(+\infty)(1-x) & 0 \\ 0 & 0 & 0 & 1\end{array}\right]$ for $x \in[0,1]$.

The conditions a) and b) come directly from (i) and (ii). Condition c) is seen to be equivalent to (iii) by writing $W_{s}\left(A_{\tau}\right)$ as the operator

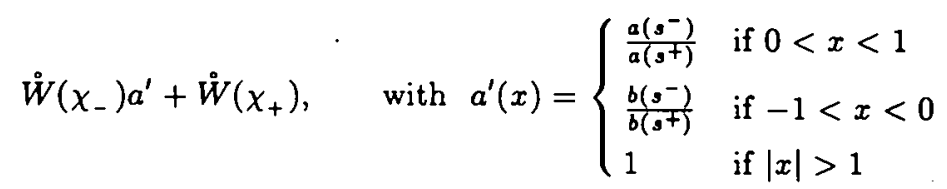

and applying usual invertibility conditions for singular integral operators (see [11]). Finally, the invertibility of $A$ implies the invertibility of $M\left(A_{\tau}\right)$

3.2 Wiener-Hopf plus Hankel operators. Now let $A$ be the operator

$$
A=\chi_{+} \stackrel{\circ}{W}(a) \chi_{+}+\chi_{+} \dot{W}(b) J \chi_{+}+\chi_{-}
$$

with $a, b \in P C(\dot{\mathbb{R}})$. Before giving the result, we must first introduce some notation.

For any pair of complex numbers $\xi$ and $\eta$ define the set $\Omega(\xi, \eta)$ in the following way. Let $r_{\xi}$ and $r_{\eta}$. denote the half-lines starting at the origin and passing through the points $-i \xi$ and $-i \eta$, respectively. If these two half-lines coincide, then $\Omega(\xi, \eta)$ is the whole complex plane except the part of $r_{\xi}$ whose points have module greater or equal than 
$2 \sqrt{|\xi \eta|}$. If they do not coincide, then consider the branch of hyperbola with $r_{\xi}$ and $r_{\eta}$ as asymptotes and that passes through the point $-i(\xi+\eta)$. This branch divides the complex plane into two components, and $\Omega(\xi, \eta)$ is the open component that contains the origin. The applicability of the finite section method for the operator $A$ is expressed in the following result.

Corollary 3.3. The approximation method (1) applies to the operator $A$ in (68) if and only if the following conditions are satisfied:

a) The operator $A$ is invertible.

b) The operator $\chi_{+} \stackrel{\circ}{W}(\tilde{a}) \chi_{+}+\chi_{-}$is invertible.

c) $b\left(0^{-}\right)-b\left(0^{+}\right) \in \Omega\left(a\left(0^{-}\right), a\left(0^{+}\right)\right)$.

d) For any $s \in \mathbb{R}^{+}$, the roots of the polynomial

$$
\xi^{2}-\left(\frac{a\left(s^{+}\right)}{a\left(s^{-}\right)}+\frac{a\left(-s^{+}\right)}{a\left(-s^{-}\right)}+\frac{b\left(s^{+}\right)-b\left(s^{-}\right)}{a\left(s^{-}\right)} \frac{b\left(-s^{+}\right)-b\left(-s^{-}\right)}{a\left(-s^{-}\right)}\right) \xi+\frac{a\left(s^{+}\right) a\left(-s^{+}\right)}{a\left(s^{-}\right) a\left(-s^{-}\right)}
$$

satisfy the inequality

$$
\left|\arg \frac{a\left(s^{+}\right)}{a\left(s^{-}\right)}+\arg \frac{a\left(-s^{+}\right)}{a\left(-s^{-}\right)}-\arg \xi\right|<\pi .
$$

Proof. The direct use of Theorem 2.37 leads to the following operators (resp. operator functions) that must be invertible to apply the approximation method:

(i) $A=\chi_{+} \stackrel{\circ}{W}(a) \chi_{+}+\chi_{+} \stackrel{\circ}{W}(b) J_{\chi_{+}}+\chi_{-}$.

(ii) $\tilde{A}=\chi_{+} \stackrel{W}{W}(\tilde{a}) \chi_{+}+\chi_{-}$.

(iii) $W_{0}\left(A_{\tau}\right)=P_{1}\left(\chi_{+}\left(a\left(0^{-}\right) \stackrel{ }{W}\left(\chi_{-}\right)+a\left(0^{+}\right) \stackrel{ }{W}\left(\chi_{+}\right)\right) \chi_{+}\right.$

$$
\left.+\chi_{+}\left(b\left(0^{-}\right) \stackrel{\circ}{W}\left(\chi_{-}\right)+b\left(0^{+}\right) \stackrel{\circ}{W}\left(\chi_{+}\right)\right) J \chi_{+}+\chi_{-}\right) P_{1}+Q_{1} \text {. }
$$

(iv) $\dot{W}_{s}\left(A_{\tau}\right)=P_{1}\left[\begin{array}{ll}S_{11} & S_{12} \\ S_{21} & S_{22}\end{array}\right] P_{1}+Q_{1}$, with

$$
\begin{aligned}
& S_{11}=\chi_{+}\left(a\left(s^{-}\right) \stackrel{\circ}{W}\left(\chi_{-}\right)+a\left(s^{+}\right) \stackrel{\circ}{W}\left(\chi_{+}\right)\right) \chi_{+}+\chi_{-} \\
& S_{12}=\chi_{+}\left(b\left(s^{-}\right) \dot{W}\left(\chi_{-}\right)+b\left(s^{+}\right) \dot{W}\left(\chi_{+}\right)\right) \chi_{-} \\
& S_{21}=\chi_{-}\left(b\left(-s^{+}\right) \dot{W}\left(\chi_{-}\right)+b\left(-s^{-}\right) \dot{W}\left(\chi_{+}\right) a b i g\right) \chi_{+} \\
& S_{22}=\chi_{-}\left(a\left(-s^{+}\right) \stackrel{\circ}{W}\left(\chi_{-}\right)+a\left(-s^{-}\right) \stackrel{\circ}{W}\left(\chi_{+}\right)\right) \chi_{-}+\chi_{+}
\end{aligned}
$$

(v) $M\left(A_{\tau}\right): x \longmapsto\left[\begin{array}{cccc}a(-\infty)(1-x)+a(+\infty) x & 0 & 0 & 0 \\ 0 & 1 & 0 & 0 \\ 0 & 0 & 1 & 0 \\ 0 & 0 & 0 & 1\end{array}\right]$ for $x \in[0,1]$. 
So conditions a) and b) come directly from (i) and (ii), and (v) is also easily seen to be satisfied if $\tilde{A}$ is invertible (condition (ii) again). Regarding (iii) and (iv), in the general setting, conditions for the invertibility of these type of operators that are both exact and easy to verify are still not known. But in the scalar case, which we are dealing here with, there can be applied the conditions obtained in [23: Theorem 4], which give directly the result stated in the corollary

\section{Relations between $A_{\tau}$ and $A$ : some notes}

4.1 Introduction. In this section we will consider $\left(A_{\tau}\right) \in \mathcal{A}^{\prime}$ as a sequence of approximation operators for the operator $A$ and try to obtain relations between the operators $A_{r}$ with $\tau$ large and the strong limit operator $A$. We will use mainly the definitions and results presented in [19]. In that reference it is presented a general idea of how to deal with problems which are of the nature of the one presented in this paper. Thus the following material can be viewed as a further concrete example where such ideias can be applied. Because of size considerations, we opted to give the results only, without proofs or comments. So the reader is advised to look at the above mentioned reference in order to fully understand this section.

Let $T$ denote the index set of elements $\{-2,-1\} \cup[0, \infty]$. Define now the *homomorphisms $\operatorname{smb}_{t}(t \in \mathrm{T})$ defined for $\left(A_{\tau}\right) \in \mathcal{A}^{\prime}$ as

$$
\operatorname{smb}_{t}\left(\left(A_{\tau}\right)\right)= \begin{cases}W^{0}\left(\left(A_{\tau}\right)\right) & \text { if } t=-2 \\ W^{1}\left(\left(A_{\tau}\right)\right) & \text { if } t=-1 \\ W_{0}\left(A_{\tau}\right) & \text { if } t=0 \\ \dot{W}_{s}\left(A_{\tau}\right) & \text { if } t \in \mathbb{R}^{+} \\ \left.M\left(A_{\tau}\right)\right) & \text { if } t=\infty\end{cases}
$$

and the algebra $\mathcal{S}$ as the algebra of functions defined on $\mathrm{T}$ and with values at $t$ in $\operatorname{Im}\left\{\mathrm{smb}_{t}\right\}$. Then we can define the homomorphism $\operatorname{smb}$ from $\mathcal{A}^{\prime} / \mathcal{G}$ to $\mathcal{S}$ by $\operatorname{smb}\left(A_{r}\right)=$ $\operatorname{smb}_{t}\left(\left(A_{\tau}\right)\right)$. This homomorphism is a symbol in the usual sense of the word and the building homomorphisms are fractal. By fractal we mean the following: Suppose that $\left(A_{r}\right)_{r \in \mathbb{R}^{+}}$is a sequence in $\mathcal{A}$ and $W$ is a homomorphism. We say that $W$ is fractal if for any infinite and unbounded index sequence $\left\{\tau_{k}\right\}_{k \in N}$ we have for the corresponding operator sequence (belonging now to an algebra of sequences indexed in the natural numbers) $W\left(A_{\tau_{k}}\right)=W\left(A_{\tau}\right)$.

For each $t \in T$ we will associate the ideal $\mathcal{I}_{t}$, with $\mathcal{I}_{-2}=\mathcal{J}_{0} \cap \mathcal{A}^{\prime}, \mathcal{I}_{-1}=\mathcal{J}_{1} \cap \mathcal{A}^{\prime}$, and $I_{t}=\{0\}$ for $t \in[0,+\infty]$. Then we have $\operatorname{smb}_{t_{1}}\left(I_{t_{2}}\right)=0$ for $t_{1} \neq t_{2}$ and that $\mathrm{smb}_{t}$ are lifting homomorphisms (i.e. $\operatorname{smb}_{t}\left(\mathcal{I}_{t}\right)$ are closed two-sided ${ }^{*}$-ideals and $\mathrm{smb}_{t}$ restricted to $\mathcal{I}_{t}$ are isomorphisms between $\mathcal{I}_{t}$ and $\operatorname{smb}_{t}\left(\mathcal{I}_{t}\right)$ ).

The two above facts, plus the realization that if the cosets $\operatorname{smb}_{t}\left(\left(A_{T}\right)\right)+\operatorname{smb}_{t}\left(\mathcal{I}_{t}\right)$ are invertible for all $t \in \mathrm{T}$, then $\left(A_{T}\right)+\mathcal{I}$ is invertible in $\mathcal{A}^{\prime} / \mathcal{I}$, where $\mathcal{I}$ is the smallest closed two-sided ideal. of $\mathcal{A}^{\prime}$ containing the ideals. $\mathcal{I}_{t}$, permit us to be in the conditions of Part 3 of the lifting theorem proved in [19], and so we obtain the following results almost immediately. 
4.2 Norms and condition numbers. Applying [19: Theorem 6] we obtain

Theorem 4.1. Let $\left(A_{\tau}\right) \in \mathcal{A}^{\prime}$. Then $\lim _{\tau \rightarrow \infty}\left\|A_{\tau}\right\|$ exists and

$$
\lim _{\tau \rightarrow \infty}\left\|A_{\tau}\right\|=\sup _{t \in T}\left\|\operatorname{smb}_{t}\left(A_{r}\right)\right\| .
$$

Moreover, if $\left(A_{\tau}\right)$ is stable, then the sequence of the condition numbers $\left\|A_{\tau}\right\|\left\|A_{\tau}^{-1}\right\|$ is convergent and

$$
\lim \left\|A_{\tau}\right\|\left\|A_{\tau}^{-1}\right\|=\sup _{t \in T}\left\|\operatorname{smb}_{t}\left(A_{\tau}\right)\right\| \sup _{t \in T}\left\|\operatorname{smb}_{t}\left(A_{T}\right)^{-1}\right\| .
$$

4.3 Moore-Penrose invertibility. We recall that an element $b$ of a $C^{*}$-algebra $\mathcal{B}$ is Moore-Penrose invertible if there is is an element $b^{\dagger}$ in $\mathcal{B}$ such that $b b^{\dagger} b=b, b^{\dagger} b b^{\dagger}=b^{\dagger}$, $\left(b b^{\dagger}\right)^{*}=b b^{\dagger}$ and $\left(b^{\dagger} b\right)^{*}=b^{\dagger} b$. An ideal of $\mathcal{B}$ is called a Moore-Penrose ideal if the invertibility of an element $b \in \mathcal{B}$ module the ideal implies always its Moore-Penrose invertibility. Note that $\operatorname{smb}_{0}\left(I^{0}\right)=\mathcal{K}$ and $\operatorname{smb}_{1}\left(I^{1}\right) \subset \mathcal{K}^{2 \times 2}$, and so $\operatorname{smb}_{t}\left(I_{t}\right)$ are Moore-Penrose ideals. Applying [19: Theorem 7 and Proposition 8] we obtain

Theorem 4.2. If the cosets $\operatorname{smb}_{t}\left(\left(A_{\tau}\right)\right)+\operatorname{smb}_{t}\left(\mathcal{I}_{t}\right)$ are invertible for all $t \in T$, then $\left(A_{\tau}\right)+\mathcal{G}$ is Moore-Pentose invertible in $(\mathcal{A}+\mathcal{G}) / \mathcal{G}$, with inverse $\left(B_{\tau}\right)+\mathcal{G}$. Moreover, any sequence in the coset $\left(B_{\tau}\right)+\mathcal{G}$ converges strongly to the Moore-Penrose inverse of $A=W^{0}\left(\left(A_{\tau}\right)\right)$.

4.4 Limiting sets of eigenvalues. Define the partial limiting set $\lim _{r \rightarrow \infty} \Omega_{\tau}$ of a sequence of subsets $\Omega_{r}$ of the complex plane as the collection of all complex numbers that for each one there exists a sequence of points $z_{\tau} \in \Omega_{\tau}$ of which they are a partial limit (sublimit). Define the uniform limiting set $\operatorname{Lim}_{\tau \rightarrow \infty} \Omega_{\tau}$ of a sequence of subsets $\Omega_{\tau}$ of the complex plane as the collection of all complex numbers that for each one there exists a sequence of points $z_{\tau} \in \Omega_{\tau}$ of which they are the limit. Then, by [19: Theorems 8 and 9 ], we have

Theorem 4.3. If $\left(A_{\tau}\right) \in \mathcal{A}^{\prime}$ is a sequence of self-adjoint operators, then

$$
\lim _{\tau \rightarrow \infty} \sigma\left(A_{\tau}\right)=\operatorname{Lim}_{\tau \rightarrow \infty} \sigma\left(A_{\tau}\right)=\bigcup_{t \in T} \sigma\left(\operatorname{smb}_{t}\left(A_{\tau}\right)\right)
$$

4.5 Limiting sets of $s$-numbers. Define the set $s(A)$ of the $s$-numbers of an operator $A$ by $s(A)=\sigma\left(\left(A^{*} A\right)^{\frac{1}{2}}\right)$, where $\left(A^{*} A\right)^{\frac{1}{2}}$ is the non-negative square root of $A^{*} A$. Then [19: Theorem 10] gives us for $\left(A_{\tau}\right) \in \mathcal{A}^{\prime}$ the following result.

Theorem 4.4. We have $\lim _{r \rightarrow \infty} s\left(A_{\tau}\right)=\operatorname{Lim}_{\tau \rightarrow \infty} s\left(A_{\tau}\right)=\bigcup_{t \in T} s\left(\operatorname{smb}_{t}\left(A_{\tau}\right)\right)$.

4.6 Limiting sets of the $\varepsilon$-pseudospectra. For $\varepsilon>0$, the $\varepsilon$-pseudospectrum of the operator $A$ is the set

$$
\sigma_{\varepsilon}(A)=\left\{z \in \mathbb{C}: z I-A \text { is not invertible or }\|z I-A\| \geq \frac{1}{\varepsilon}\right\} .
$$

Then if we apply [19: Theorem 11] (see also [1]), we obtain the following result. 
Theorem 4.5. We have $\lim _{\tau \rightarrow \infty} \sigma_{\varepsilon}\left(A_{T}\right)=\operatorname{Lim}_{\tau \rightarrow \infty} \sigma_{\varepsilon}\left(A_{\tau}\right)=\bigcup_{t \in T} \sigma_{\varepsilon}\left(\operatorname{smb}_{t}\left(A_{T}\right)\right)$.

Acknowledgment. The authors wish to acknowledge the help of Prof. F.S. Teixeira, who read the manuscript thoroughly and gave many useful suggestions to improve the presentation.

\section{References}

[1] Boettcher, A.: Pseudospectra and singular values of large convolution operators. J. Int. Equ. Appl. 6 (1995), $267-301$.

[2] Boettcher, A. and B. Silbermann: Invertibility and Asymptotics of Toeplitz Matrices. Berlin: Akademie-Verlag 1983.

[3] Boettcher, A. and B. Silbermann: The finite section method for Toeplitz operators on the quarter plane with piecewise continuous symbols. Math. Nach. 110 (1983), $279-291$.

[4] Boettcher, A. and B. Silbermann: Analysis of Toeplitz Operators. Berlin: AkademieVerlag 1989, and Berlin: Springer-Verlag 1990.

[5] Douglas, R. G.: Banach Algebra Techniques in Operator Theory. New York: Academic Press 1972.

[6] Duduchava, R.: On algebras generated by convolutions and discontinuous functions. Int. Equ. Oper. Theory 10 (1987), $505-530$.

[7] Finck, T., Roch, S. and B. Silbermann: Two projections theorems and symbol calculus for operators with massive local spectra. Math. Nach. 162 (1993), $167-185$.

[8] Gohberg, I. C. and I. A. Feldman: Convolution equations and projection methods for their solution (Transl. Math. Mon.: Vol. 41). Providence (R.I.): Amer. Math. Soc. 1974.

[9] Hagen, R., Roch, S. and B. Silbermann: Spectral Theory of Approximation Methods for Convolution Equations (Operator Theory: Vol. 74). Basel: Birkhäuser Verlag 1995.

[10] Kozak, A.: A local method in the theory of projection methods (in Russian). Dokl. Acad. Nauk SSSR 212 (1974), 1287 - 1289.

[11] Mikhlin, S.G. and S. Prössdorf: Singular Integral Operators. Berlin: Springer-Verlag 1987.

[12] Meister, E., Penzel, F., Speck, F. O. and F. S. Teixeira: Two media scattering problems in a half-space. In: Partial Differential Equations with Real Analysis (eds: H. Begehr and A. Jeffrey; Pitman Research Notes in Mathematics Series: Vol. 263). New York: Longman 1992 , pp. $122-146$.

[13] Meister, E., Penzel, F., Speck, F. O. and F. S. Teixeira: Some interior and exterior boundary value problems for the Helmholtz equation in a quadrant. Proc. Royal Soc. Edinburgh 123A (1993), 275 - 294.

[14] Penzel, F. and F. S. Teixeira: A Wiener-Hopf approach for the scattering by a disk. Int. Equ. Oper. Theory 24 (1996), $230-247$.

[15] Prössdorf S. and B. Silbermann: Numerical Analysis for Integral and Related Operator Equations. Berlin: Akademie-Verlag 1991.

[16] Roch, S.: Finite sections of operators generated by convolutions. In: Seminar Analysis, Operator Equations and Numerical Analysis 1987/88. Berlin: Karl-Weierstrass-Institut für Mathematik 1988, pp. $118-138$.

[17] Roch, S. and B. Silbermann: Algebras of convolution operators and their image in the Calkin algebra. Report. Berlin: Karl-Weierstrass-Institut für Mathematik 1990, Report R-MATH-05/90. 
[18] Roch, S. and B. Silbermann: A symbol calculus for finite sections of singular integral operators with fip and piecewise continuos coefficients. J. Funct. Anal. 78 (1988), 365 389.

[19] Roch, S. and B. Silbermann: $C^{*}$-algebra techniques in numerical analysis. J. Oper. Theory 35 (1996), $241-280$.

[20] Santos, P. A. and F. S. Teixeira: Sommerfeld half-plane problems with higher-order boundary conditions. Math. Nachr. 171 (1995), $269-282$.

[21] Silbermann, B.: Lokale Theorie des Reduktionsverfahrens für Toeplitz-Operatoren. Math. Nachr. 104 (1981), 137 - 146

[22] Silbermann, B.: Local objects in the theory of Toeplitz operators. Int. Equ. Oper. Theory 9 (1986), $706-738$.

[23] Spitkovsky, I. M. and A. M. Tashbaev: Factorization of certain piecewise constant matrix functions and its applications. Math Nachr. 151 (1991), $241-261$.

Received 30.01.1997 